JSPS Grants-in-Aid for Scientific Research (S)

Understanding Persistent Deflation in Japan

Working Paper Series

No. 081

March 2016

\title{
Parameter Bias in an Estimated DSGE Model: Does Nonlinearity Matter?
}

\author{
Yasuo Hirose \\ Takeki Sunakawa
}

UTokyo Price Project

702 Faculty of Economics, The University of Tokyo,

7-3-1 Hongo, Bunkyo-ku, Tokyo 113-0033, Japan

Tel: +81-3-5841-5595

E-mail:watlab@e.u-tokyo.ac.jp

http://www.price.e.u-tokyo.ac.jp/english/ 


\title{
Parameter Bias in an Estimated DSGE Model: Does Nonlinearity Matter?*
}

\author{
Yasuo Hirose $^{\dagger} \quad$ Takeki Sunakawa ${ }^{\ddagger}$
}

November 2015

\begin{abstract}
How can parameter estimates be biased in a dynamic stochastic general equilibrium model that omits nonlinearity in the economy? To answer this question, we simulate data from a fully nonlinear New Keynesian model with the zero lower bound constraint and estimate a linearized version of the model. Monte Carlo experiments show that significant biases are detected in the estimates of monetary policy parameters and the steady-state inflation and real interest rates. These biases arise mainly from neglecting the zero lower bound constraint rather than linearizing equilibrium conditions. With fixed parameters, the variance-covariance matrix and impulse response functions of observed variables implied by the linearized model substantially differ from those implied by its nonlinear counterpart. However, we find that the biased estimates of parameters in the estimated linear model can make most of the differences small.
\end{abstract}

Keywords: Nonlinearity, Zero lower bound, DSGE model, Parameter bias, Bayesian estimation JEL Classification: C32, E30, E52

\footnotetext{
*The authors would like to thank Toni Braun, Dario Caldara, Pablo Cuba-Borda, Jesús Fernández-Villaverde, Marc Giannoni, Anna Kormilitsina, Roberto Mariano, Taisuke Nakata, Frank Schorfheide, and participants at the NBER Workshop on Methods and Applications for DSGE Models for insightful comments and suggestions. This study forms part of the project on "Understanding Persistent Deflation in Japan" funded by a JSPS Grant-in-Aid for Scientific Research (No. 24223003).

${ }^{\dagger}$ Faculty of Economics, Keio University. E-mail: yhirose@econ.keio.ac.jp

${ }^{\ddagger}$ Graduate School of Public Policy, University of Tokyo. E-mail: sunakawa@pp.u-tokyo.ac.jp
} 


\section{Introduction}

Following the development of Bayesian estimation and evaluation techniques, many economists have estimated dynamic stochastic general equilibrium (DSGE) models using macroeconomic time series. In particular, estimated New Keynesian models, which feature nominal rigidities and monetary policy rules, have been extensively used by policy institutions such as central banks. Most of the estimated DSGE models are linearized around a steady state because a linear state-space representation along with the assumption of normality of exogenous shocks enables us to efficiently evaluate likelihood using the Kalman filter. However, Fernández-Villaverde and Rubio-Ramírez (2005) and Fernández-Villaverde, Rubio-Ramírez, and Santos (2006) demonstrate that the level of likelihood and parameter estimates based on a linearized model can be significantly different from those based on its original nonlinear model. Moreover, in the context of New Keynesian models, Basu and Bundick (2012), Braun, Körber, and Waki (2012), Fernández-Villaverde, Gordon, Guerrón-Quintana, and Rubio-Ramírez (2015), Gavin, Keen, Richter, and Throckmorton (2015), Gust, López-Salido, and Smith (2012), Nakata (2013a, 2013b), and Ngo (2014) emphasize the importance of considering nonlinearity in assessing the quantitative implications of the models when the zero lower bound (ZLB) constraint on the nominal interest rate is taken into account.

Despite the existence of these studies, attempts to estimate DSGE models in a fully nonlinear and stochastic setting are still limited. A few exceptions are Fernández-Villaverde and RubioRamírez (2005), who estimate a neoclassical growth model in a nonlinear form, and Gust, LópezSalido, and Smith (2012), who estimate a nonlinear New Keynesian DSGE model in which the ZLB constraint is occasionally binding. ${ }^{1}$ A primary reason for scarcity of such attempts is probably due to high computational costs for the estimation of nonlinear models. To evaluate the likelihood function in a nonlinear setting, one needs to rely on a nonlinear solution method and a particle filter, both of which require iterative procedures, and their computational expense grows rapidly with an increase in the dimensionality of problems.

In this paper, we examine how and to what extent parameter estimates can be biased in an estimated DSGE model when nonlinearity existing in the economy is omitted in its estimation process. If significant biases were detected, it would urge researchers to be equipped with nonlinear

\footnotetext{
${ }^{1}$ Another strand of the literature solves and estimates DSGE models approximated with higher-order perturbation methods. For instance, Fernández-Villaverde and Rubio-Ramírez (2007) estimate a real business cycle model with stochastic volatility, using a second-order perturbation method, and van Binsbergen, Fernández-Villaverde, Koijen, and Rubio-Ramírez (2012) estimate a model in which households have Epstein-Zin recursive preferences with a third-order perturbation.
} 
estimation techniques at any computational costs. Otherwise, it would at least assure practitioners that the common practice of estimating linearized models could lead to reliable estimates. To this end, we solve a fully nonlinear New Keynesian model that incorporates the ZLB constraint using a projection method and simulate artificial time series from the nonlinear model. The parameters calibrated in this data-generating process (DGP) are regarded as true values. Then, using the simulated data, a Monte Carlo experiment is conducted, in which a log-linearized version of the model is estimated without imposing the ZLB. In the estimation, we employ Bayesian methods, which are now extensively used to estimate DSGE models. We set the prior means equal to the true parameter values and assess the parameter biases arising from neglecting nonlinearity by comparing the posterior means and credible intervals with the true values. Moreover, we investigate to what extent the properties of the estimated linear model can differ from those of the true nonlinear model by comparing the variance-covariance matrices and impulse response functions of observed variables.

The analysis in this paper is an extension of Hirose and Inoue (2015), who conduct a similar experiment using the data simulated from a New Keynesian model in which nonlinearity is considered only in a monetary policy rule to incorporate the ZLB constraint but the remaining equilibrium conditions are linearized. ${ }^{2}$ While Hirose and Inoue (2015) point to parameter bias only resulting from omitting the $\mathrm{ZLB}$, the present paper is able to investigate the bias caused by missing nonlinearities regarding both the ZLB and the other equilibrium conditions.

Our main results are summarized as follows. In the baseline experiment, while the estimates of structural parameters related to preferences and nominal rigidities are not biased, significant biases are detected in the estimates of monetary policy parameters and the steady-state inflation and real interest rates. We show that these biases arise for the most part from missing the ZLB constraint rather than linearizing equilibrium conditions. Given that parameters are fixed at the true values, the variance-covariance matrix and impulse response functions of observed variables implied by the linearized model are substantially different from those implied by its nonlinear counterpart. However, we find that the linearized model with the biased estimates of parameters can successfully replicate the unconditional variances and covariances of observables generated by the true nonlinear model while some of the estimated impulse responses are noticeably different from the true ones.

\footnotetext{
${ }^{2}$ Hirose and Inoue (2015) simulate the model using the solution algorithm of Erceg and Lindé (2014) and Bodenstein, Guerrieri, and Gust (2013). In their algorithm, if the model-implied nominal interest rate falls below zero, a sequence of contractionary monetary policy shocks is added in both the current and anticipated periods so that the contemporaneous and expected interest rates at the lower bound are zero.
} 
We also demonstrate that the biases in parameter estimates increase if the true parameter values in the DGP are altered so that the frequency of binding at the ZLB and the average duration of ZLB spells increase. Nevertheless, the increased biases do not necessarily amount to larger differences in the variance-covariance matrix and impulse response functions between the estimated linear model and the true nonlinear model.

These findings have several implications for the use of the estimated linear model for macroeconomic analysis. First, the parameter estimates of the linearized model would not be biased if the ZLB were not an issue. Second, even if the ZLB affect the parameter estimates, the estimated linear model with the biased parameters can predict the volatilities and correlations of macroeconomic variables correctly to a certain extent. Third, however, researchers should be cautious in conducting a short-term simulation exercise because some of the estimated impulse response functions are substantially biased.

A technical contribution of this paper is that the DGP used in our experiment has one of the richest dynamic structures of all the New Keynesian models with the ZLB constraint solved in a fully nonlinear and stochastic setting. To make our test economy realistic enough, we incorporate habit persistence in consumption preferences, price indexation of intermediate-good firms, and monetary policy smoothing into a standard New Keynesian model. ${ }^{3}$ In solving the model, we apply an efficient algorithm for a Smolyak-based projection method developed by Judd, Maliar, Maliar, and Valero (2014) to alleviate the computational burden associated with the increased number of state variables.

The remainder of the paper proceeds as follows. Section 2 describes the model used in our analysis and a nonlinear solution method. Section 3 explains our Monte Carlo experiments and presents our results. Section 4 conducts a robustness analysis with alternative parameter settings. Section 5 is the conclusion.

\footnotetext{
${ }^{3}$ While most of the existing studies that solve nonlinear New Keynesian models with the ZLB consider models with the simplest lag structure and a limited number of shocks, Fernández-Villaverde, Gordon, Guerrón-Quintana, and Rubio-Ramírez (2015) and Maliar and Maliar (2015) solve extended models with monetary policy smoothing and a variety of structural shocks. However, their models do not incorporate consumption habit or firms' price indexation. Gust, López-Salido, and Smith (2012) consider consumption habit and monetary policy smoothing in their estimated model, but price indexation of firms is not incorporated.
} 


\section{The Model and Solution Method}

This section describes the DGP used in our analysis. The DGP is characterized by a fully nonlinear New Keynesian DSGE model with the ZLB constraint on the nominal interest rate. The nonlinear model is solved using a Smolyak algorithm in the context of a projection method.

\subsection{The Model}

In the model economy, there are households, perfectly competitive final-good firms, monopolistically competitive intermediate-good firms that face price stickiness, and a central bank. For empirical validity, the model features habit persistence in consumption preferences, price indexation to past and steady-state inflation, and monetary policy smoothing.

Each household $h \in[0,1]$ consumes final goods $C_{h, t}$, supplies labor $L_{h, t}$ to intermediate-good firms, and purchases one-period riskless bonds $B_{h, t}$ so as to maximize the utility function

$$
\mathbb{E}_{0} \sum_{t=0}^{\infty} \beta^{t}\left(\prod_{k=1}^{t} d_{k}\right)^{-1}\left[\frac{\left(C_{h, t}-\gamma C_{t-1}\right)^{1-\sigma}}{1-\sigma}-L_{h, t}\right]
$$

subject to the budget constraint

$$
P_{t} C_{h, t}+B_{h, t}=P_{t} W_{t} L_{h, t}+R_{t-1} B_{h, t-1}+T_{h, t}
$$

where $\beta \in(0,1)$ is the subjective discount factor, $\sigma>0$ is the inverse of the intertemporal elasticity of substitution, $\gamma \in[0,1]$ is the degree of external habit persistence in consumption preferences, $P_{t}$ is the price of final goods, $W_{t}$ is the real wage, $R_{t}$ is the gross nominal interest rate, and $T_{h, t}$ is the sum of a lump-sum public transfer and profits received from firms. Following Eggertsson and Woodford (2003) and Christiano, Eichenbaum, and Rebelo (2011), a shock to the discount factor $d_{t}$ affects the weight of the utility in period $t+1$ relative to the one in period $t$. The log of the discount factor shock follows an $\operatorname{AR}(1)$ process:

$$
\log d_{t}=\rho_{d} \log d_{t-1}+\varepsilon_{d, t}
$$

where $\rho_{d} \in[0,1)$ is an autoregressive coefficient and $\varepsilon_{d, t}$ is a normally distributed innovation with mean zero and standard deviation $\sigma_{d}$. The first-order conditions for optimal decisions on consumption, labor supply, and bond-holding are identical among households and therefore become

$$
\begin{aligned}
\Lambda_{t} & =\left(C_{t}-\gamma C_{t-1}\right)^{-\sigma}, \\
W_{t} & =\frac{1}{\Lambda_{t}} \\
\Lambda_{t} & =\frac{\beta}{d_{t}} \mathbb{E}_{t} \Lambda_{t+1} \frac{R_{t}^{n}}{\Pi_{t+1}}
\end{aligned}
$$


where $\Lambda_{t}$ is the marginal utility of consumption and $\Pi_{t}=P_{t} / P_{t-1}$ denotes gross inflation.

The representative final-good firm produces output $Y_{t}$ under perfect competition by choosing a combination of intermediate inputs $\left\{Y_{f, t}\right\}$ so as to maximize profit $P_{t} Y_{t}-\int_{0}^{1} P_{f, t} Y_{f, t} d f$ subject to a CES production technology $Y_{t}=\left(\int_{0}^{1} Y_{f, t}^{\frac{\theta-1}{\theta}} d f\right)^{\frac{\theta}{\theta-1}}$, where $P_{f, t}$ is the price of intermediate good $f$ and $\theta>1$ denotes the elasticity of substitution. The first-order condition for profit maximization yields the final-good firm's demand for intermediate good $f, Y_{f, t}=\left(P_{f, t} / P_{t}\right)^{-\theta} Y_{t}$, while perfect competition in the final-good market leads to $P_{t}=\left(\int_{0}^{1} P_{f, t}^{1-\theta} d f\right)^{\frac{1}{1-\theta}}$.

Each intermediate-good firm $f$ produces one kind of differentiated good $Y_{f, t}$ under monopolistic competition by choosing a cost-minimizing labor input $L_{t}$ given the real wage $W_{t}$ subject to the production function

$$
Y_{f, t}=A_{t} L_{f, t}
$$

where $A_{t}$ represents total factor productivity. The log of the productivity level follows an $\operatorname{AR}(1)$ process:

$$
\log A_{t}=\rho_{a} \log A_{t-1}+\varepsilon_{a, t}
$$

where $\rho_{a} \in[0,1)$ is an autoregressive coefficient and $\varepsilon_{a, t}$ is a normally distributed innovation with mean zero and standard deviation $\sigma_{a}$. The first-order condition for cost minimization shows that real marginal cost $M C_{t}$ is identical across intermediate-good firms and is given by

$$
M C_{t}=\frac{W_{t}}{A_{t}}
$$

In the face of the final-good firm's demand and marginal cost, the intermediate-good firms set the prices of their products on a staggered basis as in Calvo (1983). In each period, a fraction $1-\xi \in(0,1)$ of intermediate-good firms reoptimize their prices while the remaining fraction $\xi$ indexes prices to a weighted average of past inflation $\Pi_{t-1}$ and steady-state inflation $\bar{\Pi}$. The firms that reoptimize their prices in the current period then maximize expected profit

$$
\mathbb{E}_{t} \sum_{j=0}^{\infty} \xi^{j} \beta^{j}\left(\prod_{k=1}^{j} d_{k}\right)^{-1} \frac{\Lambda_{t+j}}{\Lambda_{t}}\left[\frac{P_{f, t}}{P_{t+j}} \prod_{k=1}^{j}\left(\Pi_{t+k-1}^{\iota} \bar{\Pi}^{1-\iota}\right)-M C_{t+j}\right] Y_{f, t+j}
$$

subject to the final-good firm's demand

$$
Y_{f, t+j}=\left[\frac{P_{f, t}}{P_{t+j}} \prod_{k=1}^{j}\left(\Pi_{t+k-1}^{\iota} \bar{\Pi}^{1-\iota}\right)\right]^{-\theta} Y_{t+j},
$$

where $\iota \in[0,1)$ denotes the weight of price indexation to past inflation relative to steady-state 
inflation. The first-order condition for the reoptimized price $P_{t}^{o}$ is given by

$$
\frac{P_{t}^{o}}{P_{t}}=\frac{\theta}{\theta-1} \frac{\mathbb{E}_{t} \sum_{j=0}^{\infty} \xi^{j} \beta^{j}\left(\prod_{k=1}^{j} d_{k}\right)^{-1} \frac{\Lambda_{t+j}}{\Lambda_{t}}\left[\left(\prod_{k=1}^{j}\left[\left(\frac{\Pi_{t+k-1}}{\bar{\Pi}}\right)^{\iota} \frac{\bar{\Pi}}{\Pi_{t+k}}\right]\right)^{-\theta} M C_{t+j} Y_{t+j}\right]}{\mathbb{E}_{t} \sum_{j=0}^{\infty} \xi^{j} \beta^{j}\left(\prod_{k=1}^{j} d_{k}\right)^{-1} \frac{\Lambda_{t+j}}{\Lambda_{t}}\left[\left(\prod_{k=1}^{j}\left[\left(\frac{\Pi_{t+k-1}}{\bar{\Pi}}\right)^{\iota} \frac{\bar{\Pi}}{\Pi_{t+k}}\right]\right)^{1-\theta} Y_{t+j}\right]} .
$$

Let $S_{t}=\theta \mathbb{E}_{t} \sum_{j=0}^{\infty} \xi^{j} \beta^{j}\left(\prod_{k=1}^{j} d_{k}\right)^{-1} \frac{\Lambda_{t+j}}{\Lambda_{t}}\left[\left(\prod_{k=1}^{j}\left[\left(\frac{\Pi_{t+k-1}}{\bar{\Pi}}\right)^{\iota} \frac{\bar{\Pi}}{\Pi_{t+k}}\right]\right)^{-\theta} M C_{t+j} Y_{t+j}\right]$ and $F_{t}=(\theta-$ 1) $\mathbb{E}_{t} \sum_{j=0}^{\infty} \xi^{j} \beta^{j}\left(\prod_{k=1}^{j} d_{k}\right)^{-1} \frac{\Lambda_{t+j}}{\Lambda_{t}}\left[\left(\prod_{k=1}^{j}\left[\left(\frac{\Pi_{t+k-1}}{\bar{\Pi}}\right)^{\iota} \frac{\bar{\Pi}}{\Pi_{t+k}}\right]\right)^{1-\theta} Y_{t+j}\right]$, so that $P_{t}^{o} / P_{t}=S_{t} / F_{t}$. Then, $S_{t}$ and $F_{t}$ can be written in the following recursive forms:

$$
\begin{aligned}
& S_{t}=\theta M C_{t} Y_{t}+\xi \frac{\beta}{d_{t}} \mathbb{E}_{t} \frac{\Lambda_{t+1}}{\Lambda_{t}}\left[\left(\frac{\Pi_{t+1}}{\bar{\Pi}}\right)\left(\frac{\Pi_{t}}{\bar{\Pi}}\right)^{-\iota}\right]^{\theta} S_{t+1}, \\
& F_{t}=(\theta-1) Y_{t}+\xi \frac{\beta}{d_{t}} \mathbb{E}_{t} \frac{\Lambda_{t+1}}{\Lambda_{t}}\left[\left(\frac{\Pi_{t+1}}{\bar{\Pi}}\right)\left(\frac{\Pi_{t}}{\bar{\Pi}}\right)^{-\iota}\right]^{\theta-1} F_{t+1} .
\end{aligned}
$$

Moreover, the final-good's price $P_{t}=\left(\int_{0}^{1} P_{f, t}^{1-\theta} d f\right)^{\frac{1}{1-\theta}}$ can be rewritten as

$$
1=(1-\xi)\left(\frac{S_{t}}{F_{t}}\right)^{1-\theta}+\xi\left(\frac{\Pi_{t}}{\bar{\Pi}}\right)^{\theta-1}\left(\frac{\Pi_{t-1}}{\bar{\Pi}}\right)^{-\iota(\theta-1)} .
$$

The final-good market clearing condition is

$$
Y_{t}=C_{t},
$$

while the labor market clearing condition leads to

$$
\frac{Y_{t} \Delta_{t}}{A_{t}}=\int_{0}^{1} L_{f, t} d f=L_{t}
$$

where $\Delta_{t}=\int_{0}^{1}\left(P_{f, t} / P_{t}\right)^{-\theta} d f$ represents price dispersion across the intermediate-good firms. Under the present pricing rule, the price dispersion evolves according to

$$
\Delta_{t}=(1-\xi)\left(\frac{S_{t}}{F_{t}}\right)^{-\theta}+\xi\left(\frac{\Pi_{t}}{\bar{\Pi}}\right)^{\theta}\left(\frac{\Pi_{t-1}}{\bar{\Pi}}\right)^{-\iota \theta} \Delta_{t-1} .
$$

A monetary policy rule is specified as

$$
R_{t}=\max \left[R_{t}^{*}, 1\right]
$$

where

$$
R_{t}^{*}=\left(R_{t-1}^{*}\right)^{\phi_{r}}\left[\bar{R}\left(\frac{\Pi_{t}}{\bar{\Pi}}\right)^{\phi_{\pi}}\left(\frac{Y_{t}}{\bar{Y}}\right)^{\phi_{y}}\right]^{1-\phi_{r}} \exp \left(\varepsilon_{r, t}\right)
$$


$R_{t}^{*}$ denotes the hypothetical nominal interest rate that the central bank would set according to a Taylor (1993) type monetary policy rule, where $\phi_{r} \in[0,1)$ is the policy-smoothing parameter, $\phi_{\pi} \geq 0$ and $\phi_{y} \geq 0$ are the degrees of interest rate policy response to inflation and output, and $\bar{R}$ and $\bar{Y}$ are the steady-state gross nominal interest rate and output. $\varepsilon_{r, t}$ is a monetary policy shock, which is normally distributed with mean zero and standard deviation $\sigma_{r} . R_{t}=R_{t}^{*}$ if $R_{t}^{*} \geq 1$ or the ZLB is not imposed. In the case of $R_{t}^{*}<1, R_{t}=1$ because the gross nominal interest rate cannot be lower than one.

An equilibrium is given by the sequence $\left\{Y_{t}, C_{t}, W_{t}, L_{t}, \Lambda_{t}, M C_{t}, S_{t}, F_{t}, \Pi_{t}, \Delta_{t}, R_{t}, R_{t}^{*}, d_{t}, A_{t}\right\}_{t=0}^{\infty}$ satisfying the equilibrium conditions (1)-(14).

\subsection{Parameter Setting}

The model is parameterized according to a standard choice in the literature to make our test economy as representative as possible.

The inverse of the intertemporal elasticity of substitution $\sigma$ is set at 1.5. The habit persistence and price indexation parameters $(\gamma, \iota)$ are both set at 0.5 . These three values follow from the prior means used in Smets and Wouters (2007). As is often calibrated in the New Keynesian literature, we set the Calvo parameter $\xi=0.75$, which implies that the average duration of prices is four quarters.

The monetary policy parameters $\left(\phi_{\pi}=2.0, \phi_{y}=0.5\right)$ that represent the degrees of interest rate response to inflation and output are larger than the coefficients in the original Taylor (1993) rule, reflecting the recent literature where the estimation of New Keynesian DSGE models results in the estimates of interest rate responses to inflation larger than $1.5 .^{4}$ Moreover, the larger degrees of the policy response are required to ensure the convergence of the nonlinear solution for broader sets of model parameters. The policy-smoothing parameter $\phi_{r}$ is set at 0.5 .

The steady-state gross inflation rate $\Pi$ is set at 1.005 , so that the central bank's target inflation rate is two percent annually. The inverse of the subjective discount factor $\beta$, which is equivalent to the steady-state gross real interest rate, is set at 1.0025, following the prior mean in Smets and Wouters (2007). The resulting steady-state nominal interest rate is three percent annually. This is almost the same as an average of the three-month Treasury bill rate in the post-1990 US sample.

The $\operatorname{AR}(1)$ coefficients for the shocks to the discount factor and productivity $\left(\rho_{d}, \rho_{a}\right)$ are both

\footnotetext{
${ }^{4}$ See, for instance, Lubik and Schorfheide (2004), Smets and Wouters (2007), and Justiniano, Primiceri, and Tambalotti (2010).
} 
set at 0.7. The standard deviations of these shocks $\left(\sigma_{d}, \sigma_{a}\right)$ are both 0.003 while a relatively small value is assigned to that of the monetary policy shock $\left(\sigma_{r}=0.001\right)$. These parameter values are determined so that the nominal interest rate is bounded at zero for substantial periods in a simulated sample.

\subsection{Solution Method}

The model has four endogenous state variables (lagged output $Y_{-1}$, the inflation rate $\Pi_{-1}$, the hypothetical nominal interest rate $R_{-1}^{*}$, and price dispersion $\Delta_{-1}$ ) and three exogenous shocks (the discount factor shock $d$, the productivity shock $A$, and the monetary policy shock $\left.\varepsilon_{r}\right) .{ }^{5}$ The policy functions satisfying the equilibrium conditions can be written as

$$
\mathbb{S}=h\left(\mathbb{S}_{-1}, \tau\right),
$$

where $\mathbb{S}_{-1}=\left(Y_{-1}, \Pi_{-1}, R_{-1}^{*}, \Delta_{-1}\right)$ and $\tau=\left(d, A, \varepsilon_{r}\right)$.

The policy functions are computed using a time-iteration method with a Smolyak algorithm in the context of a projection method. Below is a brief description of the computational procedure, and the details are explained in the Appendix. To solve for the policy functions on each grid point of the state space $\left(\mathbb{S}_{-1}, \tau\right)$, first we approximate each $\mathrm{AR}(1)$ process of the exogenous shocks $\tau$ using Markov chains. For each individual shock process, we use the method proposed by Rouwenhorst (1995) to determine the grid points and transition matrix for the Markov chain. Seven discrete values are chosen for the discount factor and productivity shocks, and five values are chosen for the monetary policy shock, so that the total number of grid points for the exogenous variables is $N_{\tau}=245$.

Given the exogenous shock processes, we solve for the policy functions $h\left(\mathbb{S}_{-1}, \tau\right)$ using a Smolyak algorithm as in Fernández-Villaverde, Gordon, Guerrón-Quintana, and Rubio-Ramírez (2015). ${ }^{6}$ While Fernández-Villaverde, Gordon, Guerrón-Quintana, and Rubio-Ramírez (2015) use a standard Smolyak method laid out in Malin, Krueger, and Kubler (2011), we apply a more efficient algorithm developed by Judd, Maliar, Maliar, and Valero (2014). Specifically, we construct the unidimensional disjoint sets of grid points of the endogenous state variables $\mathbb{S}_{-1}$ instead of the conventional nested sets in order to avoid repetitions of grid points. Then, we compute projection functions onto the

\footnotetext{
${ }^{5}$ Time subscripts are omitted in this section and in the Appendix as the policy functions are time-independent objects.

${ }^{6}$ Gust, López-Salido, and Smith (2012) use a tensor product of Chebyshev basis functions, as they have only three endogenous state variables by assuming Rotemberg pricing instead of Calvo pricing for modeling price stickiness.
} 
grid points with interpolation coefficients and a Chebyshev family of orthogonal basis functions. ${ }^{7}$ The grid points obtained by this algorithm are sparse; therefore the algorithm is more likely to be free from the curse of dimensionality. We use the level of approximation $\mu=2$ so that the total number of grid points is $N=41 .^{8}$ This number is much smaller compared with the one obtained by a product of the maximum number of grid points in each dimension, $\tilde{N}=5^{4}=625$. The Appendix shows that our solution method is very accurate, albeit with the reduced number of grid points.

As in Gust, López-Salido, and Smith (2012), we do not approximate $h\left(\mathbb{S}, \tau^{\prime}\right)$ directly, as the ZLB on the nominal interest rate generates kinks in the policy functions. Instead, we introduce an indicator function regarding the interest-rate regime and changes of variables so that the policy functions are smoother and easier to converge.

\section{Monte Carlo Experiments}

In this section, we conduct Monte Carlo experiments to examine how and to what extent parameter estimates can be biased if nonlinearity is omitted in estimation. Then, we analyze how parameter biases affect implications of the model by comparing the properties of the estimated linear model with those of the true nonlinear model.

\subsection{Design}

Our Monte Carlo experiments proceed as follows. First, we generate an artificial time series of output (deviation from the steady state), inflation, and the nominal interest rate from the DGP as described in the previous section, given the parameter values presented in Section 2.2. Thus, the simulated data can be regarded as the ones that reflect the nonlinearity of the model economy including the ZLB constraint on the nominal interest rate. Each simulated sample consists of 200 observations, ${ }^{9}$ corresponding to quarterly observations of 50 years. This sample size is chosen because it is comparable to the size with which many researchers estimate DSGE models in practice.

\footnotetext{
${ }^{7}$ To obtain the interpolation coefficients, we follow Judd, Maliar, Maliar, and Valero (2014) and use a Lagrange interpolation method, while Malin, Krueger, and Kubler (2011) use the closed-form formula to obtain approximated functions.

${ }^{8}$ The level of approximation $\mu=2$ is also used in Fernández-Villaverde, Gordon, Guerrón-Quintana, and RubioRamírez (2015). They document that their algorithm fails to converge with their benchmark parameters when they increase $\mu$ from 2 to 3 .

${ }^{9}$ The model is simulated for 250 periods, and the first 50 observations are eliminated.
} 
Next, using the simulated data, we estimate a linearized version of the model. Log-linearizing the equilibrium conditions (1)-(14) around the deterministic steady state and rearranging the resulting equations yields

$$
\begin{aligned}
& \tilde{Y}_{t}=\frac{1}{1+\gamma} \mathbb{E}_{t} \tilde{Y}_{t+1}+\frac{\gamma}{1+\gamma} \tilde{Y}_{t-1}-\frac{1-\gamma}{\sigma(1+\gamma)}\left(\tilde{R}_{t}-\mathbb{E}_{t} \tilde{\Pi}_{t+1}-\tilde{d}_{t}\right), \\
& \tilde{\Pi}_{t}=\frac{\beta}{1+\beta \iota} \mathbb{E}_{t} \tilde{\Pi}_{t+1}+\frac{\iota}{1+\beta \iota} \tilde{\Pi}_{t-1}+\frac{(1-\xi)(1-\xi \beta)}{\xi(1+\beta \iota)}\left[\frac{\sigma}{1-\gamma} \tilde{Y}_{t}-\frac{\sigma \gamma}{1-\gamma} \tilde{Y}_{t-1}-\tilde{A}_{t}\right], \\
& \tilde{R}_{t}=\phi_{r} \tilde{R}_{t-1}+\left(1-\phi_{r}\right)\left(\phi_{\pi} \tilde{\Pi}_{t}+\phi_{y} \tilde{Y}_{t}\right)+\varepsilon_{r, t},
\end{aligned}
$$

where the variables with represent percentage deviations from their steady-state values. From the simulated data, the percentage deviations of output from the steady state $100 \log \left(Y_{t} / \bar{Y}\right)$, the inflation rate $100 \log \Pi_{t}$, and the nominal interest rate $100 \log R_{t}$ are assumed to be observable. Then, the observation equations are

$$
\left[\begin{array}{c}
100 \log \left(Y_{t} / \bar{Y}\right) \\
100 \log \Pi_{t} \\
100 \log R_{t}
\end{array}\right]=\left[\begin{array}{c}
0 \\
\bar{\pi} \\
\overline{r r}+\bar{\pi}
\end{array}\right]+\left[\begin{array}{c}
\tilde{Y}_{t} \\
\tilde{\Pi}_{t} \\
\tilde{R}_{t}
\end{array}\right]
$$

where $\bar{\pi}=100 \log \bar{\Pi}$ and $\bar{r} r=100 \log (1 / \beta)$ are the steady-state inflation rate and the real interest rate in percentage terms, respectively. In the subsequent analysis, $\bar{\pi}$ and $\bar{r}$ are regarded as parameters to be estimated, instead of $\bar{\Pi}$ and $\beta$.

In the estimation, we employ Bayesian methods. The prior distributions of parameters are presented in the second to fourth columns of Table 1. Each prior mean is set at the corresponding true parameter value used in the DGP. Gamma distributions with a standard deviation of 0.2 are chosen for the inverse of the intertemporal elasticity of substitution $\sigma$, the monetary policy reaction parameters $\left(\phi_{\pi}, \phi_{y}\right)$, and the steady-state inflation and real interest rates $(\bar{\pi}, \bar{r} r)$. Beta distributions with a standard deviation of 0.2 are used for habit persistence $\gamma$, the Calvo parameter $\xi$, price indexation $\iota$, the policy-smoothing parameter $\phi_{r}$, and the autoregressive coefficients $\left(\rho_{d}, \rho_{a}\right)$. For the standard deviations of the shocks $\left(100 \sigma_{d}, 100 \sigma_{a}, 100 \sigma_{r}\right)$, inverse-gamma distributions with a standard deviation of 2 are assigned. To obtain the posterior distributions, we generate 200,000 draws using the random-walk Metropolis-Hastings algorithm and discard the first 25 percent of these draws. ${ }^{10}$

These steps are replicated 200 times, and the posterior means and Bayesian credible intervals for estimated parameters are averaged over the replications. Then, we can evaluate the parameter

\footnotetext{
${ }^{10}$ The scale factor for the jumping distribution in the Metropolis-Hastings algorithm is adjusted so that the acceptance rate of candidate draws is approximately 25 percent.
} 
biases arising from missing nonlinearity by examining how the resulting posterior means and credible intervals differ from the true parameter values.

According to the simulated sample of 40,000 periods (200 Monte Carlo replications with a sample size of 200 observations), the economy is at the ZLB for 9.7 percent of quarters, and the average duration of ZLB spells is 2.8 quarters. ${ }^{11}$ Compared with the simulation results in the previous studies that employ nonlinear New Keynesian models similar to ours, these statistics indicate that our model economy is more frequently constrained by the ZLB and that the average duration of ZLB spells is comparable. Fernández-Villaverde, Gordon, Guerrón-Quintana, and Rubio-Ramírez (2015) simulate a model calibrated for the US economy and show that the economy spends 5.5 percent of quarters at the ZLB and that the average duration at the ZLB is 2.1 quarters. Gust, López-Salido, and Smith (2012) estimate a model in a fully nonlinear setting using the US data from 1983:Q1 to 2011:Q4, and the simulation of their estimated model demonstrates that the economy is at the ZLB for 3.1 percent of quarters and that the average duration of the ZLB spells is about three quarters. ${ }^{12}$

\section{$3.2 \quad$ Results}

The fifth and sixth columns of Table 1 (Baseline) present averages of the posterior means and 90 percent credible intervals for estimated parameters in the baseline experiment.

The estimates of the structural parameters $(\sigma, \gamma, \xi, \iota)$, which are related to preferences and nominal rigidities, are not biased as the posterior mean estimates are very close to the true parameter values and the 90 percent credible intervals contain the true values. The policy-smoothing parameter $\phi_{r}$, the autoregressive coefficients $\left(\rho_{d}, \rho_{a}\right)$, and the standard deviations of the shocks $\left(\sigma_{d}, \sigma_{a}, \sigma_{r}\right)$ are also not affected substantially by omitting nonlinearity in the estimation.

However, we detect significant biases in the estimates of the parameters that characterize the degree of a monetary policy response to inflation $\phi_{\pi}$ and the steady-state inflation and real interest rates $(\bar{\pi}, \overline{r r})$, in the sense that the mean estimates of these parameters noticeably differ from the

\footnotetext{
${ }^{11}$ We acknowledge that this duration is too short, compared with the recent experience that major central banks have faced the ZLB for a prolonged period. We tried to parameterize the DGP so that the duration was longer, but then our solution frequently failed to converge. The previous studies referred in the subsequent sentences seem to be subject to the same issue.

${ }^{12}$ Gust, López-Salido, and Smith (2012) also document that financial market participants in 2009:Q1 expected a duration of three quarters, on average, for which the federal funds rate would remain at virtually zero, and argue that their model's median forecast of the duration conditional on the state variables in 2009:Q1 is in line with the market expectation.
} 
corresponding true values and that the credible intervals do not include the true values. The estimated monetary policy response to output $\phi_{y}$ is also biased although its credible interval contains the true value.

For the biased estimates of the monetary policy reaction parameters $\left(\phi_{\pi}, \phi_{y}\right)$, the same explanation as in Hirose and Inoue (2015) applies. In the DGP, the monetary policy reaction function has a kink where the ZLB constraint becomes binding; that is, the reaction function has positive slopes with respect to inflation and output if the unconstrained nominal interest rate is positive, but the slopes become flat if it turns negative. However, when such a kink is omitted in the estimation, as is the case in our experiment, the estimated slopes are approximated to lie between the positive and flat slopes, and thus these monetary policy parameters can be underestimated.

The downward biases detected in the estimates of the steady-state inflation and real interest rates $(\bar{\pi}, \overline{r r})$ are in stark contrast to the result in Hirose and Inoue (2015), who find upward bias in these parameters. ${ }^{13}$ In the present analysis, the DGP is characterized by the fully nonlinear model. In such an environment, the means of the ergodic distributions of endogenous variables can be substantially different from the deterministic steady-state values. In particular, once the ZLB constraint is taken into account, the presence of uncertainty can reduce inflation and output by a substantial amount, as emphasized by Nakata (2013a), and hence their levels become lower than the deterministic ones. In reality, the mean of the inflation rate simulated from the DGP, which asymptotically converges to the mean of its ergodic distribution, is 0.39 percent while the corresponding deterministic value is calibrated at 0.5. Because the latter is treated as the true value, the discrepancy between the two steady states is adjusted by the lower estimate of $\bar{\pi}$ in our experiment. The ergodic mean of the nominal interest rate can be either larger or smaller than its deterministic steady-state value. It will be larger if the direct effect of the ZLB constraint is strong. It will be smaller if the feed-back effect of reduced inflation and output on the nominal interest rate through the Taylor-type monetary policy rule is dominant. In the present experiment, the mean of the nominal interest rate simulated from the DGP is 0.57 percent, which is much lower than

\footnotetext{
${ }^{13}$ Hirose and Inoue (2015) explain the reason for the upward bias in the steady-state real interest rate as follows. The presence of the ZLB forces the nominal interest rate to be equal to or greater than zero. However, if the model estimation fails to consider the ZLB, the nominal interest rate can be negative, and the mean of the model-implied nominal interest rate declines. Then, the estimate of the steady-state nominal interest rate must rise to adjust the difference between the mean of the model-implied nominal interest rate and that of the corresponding series simulated from the DGP with the ZLB, given that the model is linearized around the steady state. In their experiment, such an adjustment has mostly emerged as a change in the estimate of the steady-state real interest rate, rather than that of the steady-state inflation rate.
} 
the deterministic steady-state value of 0.75 percent. Consequently, $\bar{r} r$ is underestimated while the downward bias in the estimate of $\bar{\pi}$ partially contributes to filling the gap between the mean of the simulated nominal interest rate and its deterministic steady state.

These sources of the parameter biases could potentially affect the estimates of other structural parameters that characterize the dynamic IS equation (15) and the New Keynesian Phillips curve (16) because we employ a system-based estimation approach. However, our experiment indicates that such an effect is quite limited.

\subsection{Which nonlinearity matters?}

Our baseline experiment suggests that omitting nonlinearity in estimation leads to substantial biases in parameter estimates. Which missing nonlinearity causes such biases, the ZLB constraint or nonlinear equilibrium conditions? To answer this question, we conduct two additional Monte Carlo experiments. First, the DGP in the baseline experiment is replaced with the one where the ZLB constraint is not imposed but the other nonlinearities remain unchanged. Second, we consider the DGP where the ZLB constraint is imposed but all the equilibrium conditions are linearized. The latter experiment is comparable to the analysis in Hirose and Inoue (2015) in the sense that the DGP is quasi-linear, although the solution methods are different from each other. ${ }^{14}$

The first additional experiment assesses to what extent parameter estimates can be biased if nonlinearities in the equilibrium conditions other than the ZLB constraint are ignored in the estimation. The seventh and eighth columns of Table 1 (No ZLB in DGP) presents the results. The parameter estimates are unbiased in that all the mean estimates are almost the same as the true values. This finding implies that the DGP in this experiment is very close to its linearized counterpart. Thus, omitting nonlinearity in estimation would not affect parameter estimates if the ZLB were not an issue.

The second additional experiment is to quantify the effect of omitting solely the ZLB constraint on parameter estimates. The results are shown in the last two columns of Table 1 (Quasi-linear DGP). As in the baseline experiment, nonnegligible biases are found in the estimates of the monetary policy reaction parameters $\left(\phi_{\pi}, \phi_{y}\right)$ and the steady-state inflation and real interest rates $(\bar{\pi}$, $\overline{r r})$, although the biases are smaller than those in the baseline experiment. ${ }^{15}$ Given that the effect

\footnotetext{
${ }^{14}$ In the present paper, we solve the quasi-linear model using a projection method described in the Appendix. Regarding the solution method employed in Hirose and Inoue (2015), see footnote 2.

${ }^{15}$ The smaller biases indicate that the quasi-linear DGP exhibits less nonlinearity than the fully nonlinear DGP. This is because nonlinearity is amplified by combining the ZLB constraint with the other nonlinear equilibrium
} 
of missing nonlinearity in the equilibrium conditions is virtually zero from the first experiment, we can conclude that the biases detected in the baseline experiment are mainly due to ignoring the ZLB constraint rather than linearizing the equilibrium conditions.

To gain more insight about the results, Figure 1 illustrates the policy functions of the nominal interest rate $R_{t}$, inflation $\Pi_{t}$, and output $Y_{t}$, for the range of the state variables, $R_{t-1}^{*}, \Pi_{t-1}$, and

$Y_{t-1}$ shown on each horizontal axis. Each panel compares the policy functions of the three DGPs considered above: the nonlinear model with the ZLB (thick solid line in red), the nonlinear model without the ZLB (thin solid line in blue), and the quasi-linear model with the ZLB (dashed lines in black). Two points should be stressed here. First, the policy functions of the nonlinear model without the ZLB appear to be almost linear, indicating that the linear model can approximate well the nonlinear model in the absence of the ZLB. Thus, no biases are detected in the first additional experiment. Second, the policy functions of the nonlinear model with the ZLB and those of the quasi-linear model with the ZLB are very similar to each other and exhibit strong nonlinearities in the panels associated with the nominal interest rate. This is the reason why the substantial biases are found in the estimates of the monetary policy parameters and the steady-state inflation and real interest rates both in the baseline experiment and in the second additional experiment.

\subsection{Properties of the estimated model}

From a practical perspective, it is important to investigate to what extent the properties of the estimated model that omits nonlinearity can differ from those of the true nonlinear model. To examine this issue, we compare the variance-covariance matrix and impulse response functions of the observed variables based on the estimated linear model with those based on the true nonlinear model. There are two potential sources that can alter the implications of the estimated model: linearization of the model and the biased estimates of parameters. To disentangle these two sources, the properties of the linear model with the true parameter values are also compared.

\subsubsection{Variances and covariances}

Table 2 compares the unconditional variance-covariance matrices of output, inflation, and the nominal interest rate implied by (1) the nonlinear model with the true parameters (i.e., the baseline DGP), (2) the linear model with the true parameters, and (3) the linear model with the biased parameters. In the case of the nonlinear model with the true parameters, the empirical variance-

conditions. However, we find such a difference is admittedly small. 
covariance matrix is calculated by averaging the sample variances and covariances based on the simulated data (200 Monte Carlo replications with a sample size of 200 observations) used in the baseline experiment. In the cases of the linear models with the true and biased parameters, the theoretical variance-covariance matrices are computed by solving the discrete Lyapunov equations. The biased parameters are set at the posterior mean estimates obtained in the baseline experiment.

Comparing the cases of the nonlinear and linear models, both of which are based on the true parameter values, we find that all the components of the variance-covariance matrix implied by the linear model are remarkably smaller than those implied by the nonlinear model. This is because linearizing the model eliminates the effects of the ZLB constraint and associated uncertainty, both of which could amplify the volatility of macroeconomic variables.

However, the last three rows in the table show that the linear model with the biased parameters makes the variance-covariance matrix very close to that implied by the nonlinear model with the true parameters. In this case, the monetary policy reaction parameters $\left(\phi_{\pi}, \phi_{y}\right)$ are underestimated, as presented in Section 3.2, and the less aggressive monetary policy makes the economy more volatile. Thus, even with the linearized model, the biased estimates of parameters can retrieve the variances and covariances of the observables generated by its nonlinear counterpart.

\subsubsection{Impulse responses}

Figure 2 depicts the impulse responses of output, inflation, and the nominal interest rate to one standard deviation shocks to the (1) discount factor, (2) productivity, and (3) monetary policy. ${ }^{16}$ In each panel, the thick solid (red) line represents the true response based on the nonlinear model with the true parameters (i.e., the baseline DGP), the (black) dots show the response of the linear model with the true parameters, and the thin solid (blue) and dashed (blue) lines are the posterior mean and 90 percent credible interval of the estimated response given the linear model with the biased estimates of parameters, respectively. The responses are expressed in terms of percentage deviations from the steady state in order to exclude the effects of the biases in the steady-state parameters and to focus on changes in the transmission of each shock. ${ }^{17}$

With the true parameters, sizeable differences are found between the responses of the linear

\footnotetext{
${ }^{16}$ We do not consider any shocks that cause the ZLB to bind because it is obvious for the linearized responses, which omit the ZLB, to differ from the true ones, which take account of the ZLB.

${ }^{17}$ The estimated responses of inflation and the nominal interest rates in level terms are clearly different from the true responses because the estimates of the steady-state inflation and real interest rates are substantially biased as argued in Section 3.2.
} 
model and those of the nonlinear model, in particular, regarding the shocks to the discount factor and productivity. These differences arise from the fact that the policy functions of the nonlinear model with the ZLB constraint are different from those of the linear model without the constraint. In contrast, the mean estimates of the responses to these shocks become closer to the true responses, implying that the biased parameters contribute to partially replicating the true responses to these shocks.

However, the estimated response of the interest rate to the productivity shock and those of output and inflation to the monetary policy shock are substantially different from their corresponding true responses for several periods after the shocks, in that the estimated 90 percent credible intervals no longer include the true responses. Therefore, the biased parameters in the estimated linear model are not so helpful to replicate the short-term dynamics associated with these responses characterized by the true nonlinear model.

\section{Robustness Analysis}

According to the DGP in our baseline experiment, the nominal interest rate is bounded at zero for 9.7 percent of quarters, and the average duration of ZLB spells is 2.8 quarters. As the probability of hitting the ZLB increases in the simulated sample, parameter biases due to excluding the nonlinearity in the estimation might become large. To analyze such a possibility, we change the parameters assigned to the DGP so that the model economy is more frequently constrained by the ZLB. We then conduct the same Monte Carlo exercises as the baseline experiment using the data simulated by the DGPs with the alternative parameter settings. Specifically, we consider two cases. One is where the true steady-state real interest rate $\bar{r}$ falls by 0.03 (0.12 percent annually) so that $\overline{r r}=0.22$. In this case, the probability of being stuck at the ZLB is 14.8 percent of the simulated sample. The other is where the standard deviation of the discount factor shock $100 \sigma_{d}$ increases from 0.3 to 0.33 . In the latter case, the number of zero interest rate periods increases because large negative shocks are more likely to depress the economy and to lower the nominal interest rate, and the model economy is at the ZLB for 14.2 percent of quarters. In both cases, the average duration of ZLB spells is 3.1 quarters, which is longer than that in the baseline experiment. ${ }^{18}$

\footnotetext{
${ }^{18}$ The solutions did not converge if we parameterized the DGPs so that the probability of being at the ZLB or the duration of ZLB spells increases more.
} 
Parameter estimates Table 3 presents averages of the posterior means and 90 percent credible intervals for the parameters in the two alternative experiments: the case of low $\overline{r r}$ and the case of large $\sigma_{d}$. In both cases, the biases in the estimates of the monetary policy reaction parameters $\left(\phi_{\pi}\right.$, $\left.\phi_{y}\right)$ and the steady-state inflation and real interest rates $(\bar{\pi}, \bar{r} r)$ become larger than those detected in the baseline experiment (Table 1). In particular, the credible interval for $\phi_{y}$ no longer includes the true value. Moreover, significant biases are detected in the estimates of the standard deviation of the monetary policy shock $\sigma_{r}$. The reason for the upward biases in $\sigma_{r}$ is straightforward. In the simulated data, the nominal interest rate never falls below zero because the DGP takes account of the ZLB constraint. In the estimation process, however, the model-implied nominal interest rate can be negative, and such a discrepancy must be captured by the monetary policy shock $\varepsilon_{t}^{r}$, which results in the increase in $\sigma_{r}$. The estimates of the other parameters are almost unbiased, as in the baseline experiment, and hence are robust to the increased probabilities and durations of the ZLB in the DGPs.

Variances and covariances Table 4 compares the unconditional variance-covariance matrices of output, inflation, and the nominal interest rate based on (1) the nonlinear model with the true parameters, (2) the linear model with the true parameters, and (3) the linear model with the biased parameters, in the two alternative experiments. Given the true parameter values, the differences between the variances and covariances implied by the nonlinear model and those implied by the linear model become larger in both cases than in the baseline case (Table 2). As in the baseline experiment, however, the biased parameters help to match the variance-covariance matrix based on the linear model to that based on the true nonlinear model.

Impulse responses Figure 3 and 4 show the impulse responses of output, inflation, and the nominal interest rate to each one standard deviation shock in terms of percentage deviations from the steady state in the case of low $\overline{r r}$ and the case of large $\sigma_{d}$, respectively. As in the preceding section, each panel draws the true response of the nonlinear model (thick solid line in red), the response of the linear model with true parameters (dots in black), and the posterior mean (thin solid line in blue) and 90 percent credible interval (dashed lines in blue) of the estimated response. Compared with the baseline experiment (Figure 2), the differences in the responses between the linear model with true parameters and the true nonlinear model become large throughout the figures. As for the differences between the estimated and true responses, changes in the differences are limited regarding the responses of output and inflation to the discount factor shock and the pro- 
ductivity shock in both cases. However, the widening differences are found in the other responses; in particular, the 90 percent credible intervals for the estimated response of the interest rate to the productivity shock and those of output and inflation to the monetary policy shock do not include the true responses. These findings are qualitatively the same as in the baseline experiment.

\section{Concluding Remarks}

This paper has investigated parameter bias in an estimated DSGE model that neglects nonlinearity intrinsic to the economy. According to the results in our Monte Carlo experiments, significant biases have been detected in the parameter estimates associated with the monetary policy rule and the steady-state inflation and real interest rates. These biases are caused by ignoring the ZLB constraint rather than linearizing the equilibrium conditions. We have demonstrated that the estimated linear model with the biased parameters can recover the variances and covariances of observed variables implied by the true nonlinear model. However, it should be kept in mind that some of the estimated impulse response functions can be substantially different from the true ones.

Our finding regarding the source of parameter bias indicates that omitting nonlinearity in estimation would not affect parameter estimates if the ZLB were not an issue. Also, even if the ZLB matters, the biased estimates of parameters can help a linearized model replicate the time series properties of a nonlinear DGP. However, these results might not hold if the DGP is characterized by a more highly nonlinear model than considered in this paper, such as the one with recursive preferences, state-dependent pricing, or increased uncertainty. Investigating such a possibility is left for future research. 


\section{Appendix}

To solve for the policy functions on each grid point of the state space $\left(\mathbb{S}_{-1}, \tau\right)$, we follow the approach in Christiano and Fisher (2000) and Gust, López-Salido, and Smith (2012). We consider the regime-specific policy functions $h_{i}\left(\mathbb{S}_{-1}, \tau\right)$ for $i=\{1,2\}$, whose index is associated with the interest-rate regime. Specifically, the policy functions are defined as weighted averages of the regime-specific functions:

$$
\begin{gathered}
Y=h_{Y}\left(\mathbb{S}_{-1}, \tau\right)=h_{Y, 1}\left(\mathbb{S}_{-1}, \tau\right) 1_{\left\{h_{R, 1}\left(\mathbb{S}_{-1}, \tau>1\right\}\right.}+h_{Y, 2}\left(\mathbb{S}_{-1}, \tau\right) 1_{\left\{h_{R, 1}\left(\mathbb{S}_{-1}, \tau\right) \leq 1\right\}}, \\
\Pi=h_{\Pi}\left(\mathbb{S}_{-1}, \tau\right)=h_{\Pi, 1}\left(\mathbb{S}_{-1}, \tau\right) 1_{\left\{h_{R, 1}\left(\mathbb{S}_{-1}, \tau\right)>1\right\}}+h_{\Pi, 2}\left(\mathbb{S}_{-1}, \tau\right) 1_{\left\{h_{R, 1}\left(\mathbb{S}_{-1}, \tau\right) \leq 1\right\}}, \\
\Delta=h_{\Delta}\left(\mathbb{S}_{-1}, \tau\right)=h_{\Delta, 1}\left(\mathbb{S}_{-1}, \tau\right) 1_{\left\{h_{R, 1}\left(\mathbb{S}_{-1}, \tau\right)>1\right\}}+h_{\Delta, 2}\left(\mathbb{S}_{-1}, \tau\right) 1_{\left\{h_{R, 1}\left(\mathbb{S}_{-1}, \tau\right) \leq 1\right\}}, \\
R^{*}=h_{R}\left(\mathbb{S}_{-1}, \tau\right)=h_{R, 1}\left(\mathbb{S}_{-1}, \tau\right) 1_{\left\{h_{R, 1}\left(\mathbb{S}_{-1}, \tau\right)>1\right\}}+h_{R, 2}\left(\mathbb{S}_{-1}, \tau\right) 1_{\left\{h_{R, 1}\left(\mathbb{S}_{-1}, \tau\right) \leq 1\right\}},
\end{gathered}
$$

where the regime-specific monetary policy rule is given by

$$
\begin{aligned}
& h_{R, 1}\left(\mathbb{S}_{-1}, \tau\right)=\left(R_{-1}^{*}\right)^{\phi_{r}}\left[\bar{R}\left(\frac{h_{\Pi, 1}\left(\mathbb{S}_{-1}, \tau\right)}{\bar{\Pi}}\right)^{\phi_{\pi}}\left(\frac{h_{Y, 1}\left(\mathbb{S}_{-1}, \tau\right)}{\bar{Y}}\right)^{\phi_{y}}\right]^{1-\phi_{r}} \exp \left(\varepsilon_{r}\right), \\
& h_{R, 2}\left(\mathbb{S}_{-1}, \tau\right)=1,
\end{aligned}
$$

and $1_{\{D\}}$ is the indicator function that is one if the condition $D$ is true and zero otherwise. The hypothetical nominal interest rate, $R^{*}$, is either above or below the lower bound of $\bar{R}=1$.

Given the processes of $\tau$, the regime-specific functions in each regime $i=1,2$ satisfy the following equilibrium conditions:

$$
\begin{aligned}
\frac{1}{\mathcal{W}_{i}\left(\mathbb{S}_{-1}, \tau\right)} & =\beta d^{-1} h_{R, i}\left(\mathbb{S}_{-1}, \tau\right) \sum_{\tau^{\prime}}\left\{\frac{\Phi\left(\tau^{\prime} \mid \tau\right)}{\mathcal{W}\left(\mathbb{S}, \tau^{\prime}\right) h_{\Pi}\left(\mathbb{S}, \tau^{\prime}\right)}\right\} \\
S_{i}\left(\mathbb{S}_{-1}, \tau\right) & =\theta \mathcal{W}_{i}\left(\mathbb{S}_{-1}, \tau\right) A^{-1} \\
& +\beta d^{-1} \xi \sum_{\tau^{\prime}} \Phi\left(\tau^{\prime} \mid \tau\right)\left\{\left[\left(\frac{h_{\Pi}\left(\mathbb{S}, \tau^{\prime}\right)}{\bar{\Pi}}\right)\left(\frac{h_{\Pi, i}\left(\mathbb{S}_{-1}, \tau\right)}{\bar{\Pi}}\right)^{-\iota}\right]^{\theta} \frac{\mathcal{W}_{i}\left(\mathbb{S}_{-1}, \tau\right)}{\mathcal{W}\left(\mathbb{S}_{,} \tau^{\prime}\right)} \frac{h_{Y}\left(\mathbb{S}_{,} \tau^{\prime}\right)}{h_{Y, i}\left(\mathbb{S}_{-1}, \tau\right)} S\left(\mathbb{S}, \tau^{\prime}\right)\right\} \\
F_{i}\left(\mathbb{S}_{-1}, \tau\right) & =(\theta-1) \\
& +\beta d^{-1} \xi \sum_{\tau^{\prime}} \Phi\left(\tau^{\prime} \mid \tau\right)\left\{\left[\left(\frac{h_{\Pi}\left(\mathbb{S}, \tau^{\prime}\right)}{\bar{\Pi}}\right)\left(\frac{h_{\Pi, i}\left(\mathbb{S}_{-1}, \tau\right)}{\bar{\Pi}}\right)^{-\iota}\right]^{\theta-1} \frac{\mathcal{W}_{i}\left(\mathbb{S}_{-1}, \tau\right)}{\mathcal{W}\left(\mathbb{S}_{,} \tau^{\prime}\right)} \frac{h_{Y}\left(\mathbb{S}_{,} \tau^{\prime}\right)}{h_{Y, i}\left(\mathbb{S}_{-1}, \tau\right)} F\left(\mathbb{S}, \tau^{\prime}\right)\right\} \\
1 & =(1-\xi)\left(\frac{S_{i}\left(\mathbb{S}_{-1}, \tau\right)}{F_{i}\left(\mathbb{S}_{-1}, \tau\right)}\right)^{1-\theta}+\xi\left[\left(\frac{h_{\Pi, i}\left(\mathbb{S}_{-1}, \tau\right)}{\bar{\Pi}}\right)\left(\frac{\Pi-1}{\bar{\Pi}}\right)^{-\iota}\right]^{\theta-1}, \\
h_{\Delta, i}\left(\mathbb{S}_{-1}, \tau\right) & =(1-\xi)\left(\frac{S_{i}\left(\mathbb{S}_{-1}, \tau\right)}{F_{i}\left(\mathbb{S}_{-1}, \tau\right)}\right)^{-\theta}+\xi\left[\left(\frac{h_{\Pi, i}\left(\mathbb{S}_{-1}, \tau\right)}{\bar{\Pi}}\right)\left(\frac{\Pi-1}{\bar{\Pi}}\right)^{-\iota}\right]^{\theta} \Delta_{-1},
\end{aligned}
$$


where $\mathcal{W}_{i}\left(\mathbb{S}_{-1}, \tau\right) \equiv\left(h_{Y, i}\left(\mathbb{S}_{-1}, \tau\right)-\gamma Y_{-1}\right)^{\sigma}, \Phi\left(\tau^{\prime} \mid \tau\right)$ is a transition probability from $\tau$ to $\tau^{\prime}$, and $h_{\Pi}\left(\mathbb{S}, \tau^{\prime}\right), h_{Y}\left(\mathbb{S}, \tau^{\prime}\right), S\left(\mathbb{S}, \tau^{\prime}\right), F\left(\mathbb{S}, \tau^{\prime}\right)$ and $\mathcal{W}\left(\mathbb{S}, \tau^{\prime}\right)$ are weighted averages of the regime-specific functions by the indicator functions in the next period. ${ }^{19}$

Furthermore, as in Gust, López-Salido, and Smith (2012), we apply the following changes of variables:

$$
\begin{aligned}
& \mathcal{V}_{i}\left(\mathbb{S}_{-1}, \tau\right) \equiv \sum_{\tau^{\prime}} \Phi\left(\tau^{\prime} \mid \tau\right)\left\{\left[\left(\frac{h_{\Pi}\left(\mathbb{S}, \tau^{\prime}\right)}{\bar{\Pi}}\right)\left(\frac{h_{\Pi, i}\left(\mathbb{S}_{-1}, \tau\right)}{\bar{\Pi}}\right)^{-\iota}\right]^{\theta} \frac{h_{Y}\left(\mathbb{S}, \tau^{\prime}\right) S\left(\mathbb{S}, \tau^{\prime}\right)}{\mathcal{W}\left(\mathbb{S}_{-1}, \tau\right) h_{Y, i}\left(\mathbb{S}_{-1}, \tau\right)}\right\}, \\
& \mathcal{K}_{i}\left(\mathbb{S}_{-1}, \tau\right) \equiv \sum_{\tau^{\prime}} \Phi\left(\tau^{\prime} \mid \tau\right)\left\{\left[\left(\frac{h_{\Pi}\left(\mathbb{S}, \tau^{\prime}\right)}{\bar{\Pi}}\right)\left(\frac{h_{\Pi, i}\left(\mathbb{S}_{-1}, \tau\right)}{\bar{\Pi}}\right)^{-\iota}\right]^{\theta-1} \frac{h_{Y}\left(\mathbb{S}, \tau^{\prime}\right) F\left(\mathbb{S}, \tau^{\prime}\right)}{\mathcal{W}\left(\mathbb{S}_{-1}, \tau\right) h_{Y, i}\left(\mathbb{S}_{-1}, \tau\right)}\right\} .
\end{aligned}
$$

We employ the policy function iteration algorithm with initial values of $\mathcal{W}_{i}^{(0)}\left(\mathbb{S}_{-1}, \tau\right), \mathcal{V}_{i}^{(0)}\left(\mathbb{S}_{-1}, \tau\right)$ and $\mathcal{K}_{i}^{(0)}\left(\mathbb{S}_{-1}, \tau\right)$ on the grid points. The values of $S_{i}\left(\mathbb{S}_{-1}, \tau\right)$ and $F_{i}\left(\mathbb{S}_{-1}, \tau\right)$ are obtained from the values of $\mathcal{W}_{i}^{(j-1)}\left(\mathbb{S}_{-1}, \tau\right), \mathcal{V}_{i}^{(j-1)}\left(\mathbb{S}_{-1}, \tau\right)$ and $K_{i}^{(j-1)}\left(\mathbb{S}_{-1}, \tau\right)$ on the grid points in each iteration $j=1,2, \ldots{ }^{20}$ Therefore, we can solve for the policy functions $h_{i}\left(\mathbb{S}_{-1}, \tau\right)$ at each grid point without relying on any numerical solvers. As a result of the changes of variables, the previous equations become

$$
\begin{aligned}
& S_{i}\left(\mathbb{S}_{-1}, \tau\right)=\theta \mathcal{W}_{i}^{(j-1)}\left(\mathbb{S}_{-1}, \tau\right) A^{-1}+d^{-1} \beta \xi \mathcal{W}_{i}^{(j-1)}\left(\mathbb{S}_{-1}, \tau\right) \mathcal{V}_{i}^{(j-1)}\left(\mathbb{S}_{-1}, \tau\right), \\
& F_{i}\left(\mathbb{S}_{-1}, \tau\right)=(\theta-1)+d^{-1} \beta \xi \mathcal{W}_{i}^{(j-1)}\left(\mathbb{S}_{-1}, \tau\right) \mathcal{K}_{i}^{(j-1)}\left(\mathbb{S}_{-1}, \tau\right) .
\end{aligned}
$$

Furthermore, the policy functions of $h_{\Pi, i}\left(\mathbb{S}_{-1}, \tau\right)$ and $h_{Y, i}\left(\mathbb{S}_{-1}, \tau\right)$ are given by

$$
\begin{aligned}
& h_{\Pi, i}\left(\mathbb{S}_{-1}, \tau\right)=\Pi\left(\frac{\Pi_{t-1}}{\bar{\Pi}}\right)^{\iota}\left(\xi^{-1}\left[1-(1-\xi)\left(\frac{S_{i}\left(\mathbb{S}_{-1}, \tau\right)}{F_{i}\left(\mathbb{S}_{-1}, \tau\right)}\right)^{1-\theta}\right]\right)^{\frac{1}{\theta-1}} \\
& h_{Y, i}\left(\mathbb{S}_{-1}, \tau\right)=\mathcal{W}_{i}^{(j-1)}\left(\mathbb{S}_{-1}, \tau\right)^{1 / \sigma}+\gamma Y_{-1}
\end{aligned}
$$

Equations (A.1)-(A.4) imply that there is a one-to-one mapping from $\mathcal{W}_{i}^{(j-1)}\left(\mathbb{S}_{-1}, \tau\right), \mathcal{V}_{i}^{(j-1)}\left(\mathbb{S}_{-1}, \tau\right)$ and $\mathcal{K}_{i}^{(j-1)}\left(\mathbb{S}_{t-1}, \tau_{t}\right)$ to $h_{\Pi, i}\left(\mathbb{S}_{t-1}, \tau_{t}\right)$ and $h_{Y, i}\left(\mathbb{S}_{-1}, \tau\right)$. Then the values of $\mathcal{W}_{i}^{(j)}\left(\mathbb{S}_{-1}, \tau\right), \mathcal{V}_{i}^{(j)}\left(\mathbb{S}_{-1}, \tau\right)$

\footnotetext{
${ }^{19}$ Note that these weighted averaged functions are affected by the possibility that the nominal interest rate can be bounded at zero in the next period. They also depend on the values of the policy functions in the current period, $\mathbb{S}=h_{i}\left(\mathbb{S}_{-1}, \tau\right)$.

${ }^{20}$ For simplicity of notation, we omit the index $j$ from the functions except for $\mathcal{W}_{i}^{(j)}\left(\mathbb{S}_{t-1}, \tau_{t}\right), \mathcal{V}_{i}^{(j)}\left(\mathbb{S}_{t-1}, \tau_{t}\right)$ and $\mathcal{K}_{i}^{(j)}\left(\mathbb{S}_{t-1}, \tau_{t}\right)$
} 
and $\mathcal{K}_{i}^{(j)}\left(\mathbb{S}_{-1}, \tau\right)$ are updated as

$$
\begin{aligned}
\mathcal{W}_{i}^{(j)}\left(\mathbb{S}_{-1}, \tau\right) & =\left[\beta d^{-1} h_{R, i}\left(\mathbb{S}_{-1}, \tau\right) \sum_{\tau^{\prime}}\left\{\frac{\Phi\left(\tau^{\prime} \mid \tau\right)}{\hat{\mathcal{W}}\left(\mathbb{S} ; \tau^{\prime}\right) \hat{h}_{\Pi}\left(\mathbb{S} ; \tau^{\prime}\right)}\right\}\right]^{-1}, \\
\mathcal{V}_{i}^{(j)}\left(\mathbb{S}_{-1}, \tau\right) & =\sum_{\tau_{t+1}}\left\{\left[\left(\frac{\hat{h}_{\Pi}\left(\mathbb{S} ; \tau^{\prime}\right)}{\bar{\Pi}}\right)\left(\frac{h_{\Pi, i}\left(\mathbb{S}_{-1}, \tau\right)}{\bar{\Pi}}\right)^{-\iota}\right]^{\theta} \frac{\hat{h}_{Y}\left(\mathbb{S} ; \tau^{\prime}\right)}{h_{Y, i}\left(\mathbb{S}_{-1}, \tau\right)}\right. \\
& \left.\times\left(\theta A^{\prime-1}+\beta d^{\prime-1} \xi \hat{\mathcal{V}}\left(\mathbb{S} ; \tau^{\prime}\right)\right) \Phi\left(\tau^{\prime} \mid \tau\right)\right\}, \\
\mathcal{K}_{i}^{(j)}\left(\mathbb{S}_{-1}, \tau\right) & =\sum_{\tau_{t+1}}\left\{\left[\left(\frac{\hat{h}_{\Pi}\left(\mathbb{S} ; \tau^{\prime}\right)}{\bar{\Pi}}\right)\left(\frac{h_{\Pi, i}\left(\mathbb{S}_{-1}, \tau\right)}{\bar{\Pi}}\right)^{-\iota}\right]^{\theta-1} \frac{\hat{h}_{Y}\left(\mathbb{S}_{\tau^{\prime}}\right)}{h_{Y, i}\left(\mathbb{S}_{-1}, \tau\right)}\right. \\
& \left.\times\left((\theta-1) / \hat{\mathcal{W}}\left(\mathbb{S} ; \tau^{\prime}\right)+\beta d^{\prime-1} \xi \hat{\mathcal{K}}\left(\mathbb{S} ; \tau^{\prime}\right)\right) \Phi\left(\tau^{\prime} \mid \tau\right)\right\},
\end{aligned}
$$

where the estimated values of $\hat{\mathcal{W}}\left(\mathbb{S} ; \tau^{\prime}\right), \hat{\mathcal{V}}\left(\mathbb{S} ; \tau^{\prime}\right)$ and $\hat{\mathcal{K}}\left(\mathbb{S} ; \tau^{\prime}\right)$ are used to obtain the values of $\hat{h}_{\Pi}\left(\mathbb{S} ; \tau^{\prime}\right)$ and $\hat{h}_{Y}\left(\mathbb{S} ; \tau^{\prime}\right)$ from (A.1)-(A.4). To obtain the estimated values, we approximate the functions $\hat{\mathcal{W}}\left(\mathbb{S} ; \tau^{\prime}\right) \approx \mathcal{W}^{(j-1)}\left(\mathbb{S}, \tau^{\prime}\right), \hat{\mathcal{V}}\left(\mathbb{S} ; \tau^{\prime}\right) \approx \mathcal{V}^{(j-1)}\left(\mathbb{S}, \tau^{\prime}\right)$ and $\hat{\mathcal{K}}\left(\mathbb{S} ; \tau^{\prime}\right) \approx \mathcal{K}^{(j-1)}\left(\mathbb{S}, \tau^{\prime}\right)$ for any points in the domain of $\mathbb{S}$ for given $\tau^{\prime}$ by a projection method. The values of the approximated functions on the grid points $\left(\mathbb{S}_{-1}, \tau\right)$ are given by

$$
\begin{aligned}
& \mathcal{W}^{(j-1)}\left(\mathbb{S}_{-1}, \tau\right)=\mathcal{W}_{1}^{(j-1)}\left(\mathbb{S}_{-1}, \tau\right) 1_{\left\{h_{R, 1}\left(\mathbb{S}_{-1}, \tau\right)>1\right\}}+\mathcal{W}_{2}^{(j-1)}\left(\mathbb{S}_{-1}, \tau\right) 1_{\left\{h_{R, 1}\left(\mathbb{S}_{-1}, \tau\right) \leq 1\right\}} \\
& \mathcal{V}^{(j-1)}\left(\mathbb{S}_{-1}, \tau\right)=\mathcal{V}_{1}^{(j-1)}\left(\mathbb{S}_{-1}, \tau\right) 1_{\left\{h_{R, 1}\left(\mathbb{S}_{-1}, \tau\right)>1\right\}}+\mathcal{V}_{2}^{(j-1)}\left(\mathbb{S}_{-1}, \tau\right) 1_{\left\{h_{R, 1}\left(\mathbb{S}_{-1}, \tau\right) \leq 1\right\}} \\
& \mathcal{K}^{(j-1)}\left(\mathbb{S}_{-1}, \tau\right)=\mathcal{K}_{1}^{(j-1)}\left(\mathbb{S}_{-1}, \tau\right) 1_{\left\{h_{R, 1}\left(\mathbb{S}_{-1}, \tau\right)>1\right\}}+\mathcal{K}_{2}^{(j-1)}\left(\mathbb{S}_{-1}, \tau\right) 1_{\left\{h_{R, 1}\left(\mathbb{S}_{-1}, \tau\right) \leq 1\right\}}
\end{aligned}
$$

The algorithm is summarized as follows.

1. $\mathcal{W}_{i}^{(0)}\left(\mathbb{S}_{-1}, \tau\right), \mathcal{V}_{i}^{(0)}\left(\mathbb{S}_{-1}, \tau\right)$ and $\mathcal{K}_{i}^{(0)}\left(\mathbb{S}_{-1}, \tau\right)$ are given on each grid point $\left(\mathbb{S}_{-1}, \tau\right)$ as initial values.

2. $h_{i}\left(\mathbb{S}_{-1}, \tau\right)$ is computed for each regime $i=1,2$ from (A.1)-(A.4), as the values of $\mathcal{W}_{i}^{(j-1)}\left(\mathbb{S}_{-1}, \tau\right)$, $\mathcal{V}_{i}^{(j-1)}\left(\mathbb{S}_{-1}, \tau\right)$ and $\mathcal{K}_{i}^{(j-1)}\left(\mathbb{S}_{t-1}, \tau_{t}\right)$ are given on each grid point.

3. The estimated values $\hat{\mathcal{W}}\left(\mathbb{S} ; \tau^{\prime}\right), \hat{\mathcal{V}}\left(\mathbb{S} ; \tau^{\prime}\right)$ and $\hat{\mathcal{K}}\left(\mathbb{S} ; \tau^{\prime}\right)$ in the next period are obtained by a projection method.

4. $\hat{h}\left(h_{i}\left(\mathbb{S}_{-1}, \tau\right) ; \tau^{\prime}\right)$ is computed for each regime $i=1,2$ on each grid point $\left(\mathbb{S}_{-1}, \tau, \tau^{\prime}\right)$ from (A.1)-(A.4).

5. $\mathcal{W}_{i}^{(j)}\left(\mathbb{S}_{-1}, \tau\right), \mathcal{V}_{i}^{(j)}\left(\mathbb{S}_{-1}, \tau\right)$ and $\mathcal{K}_{i}^{(j)}\left(\mathbb{S}_{-1}, \tau\right)$ are updated on each grid point $\left(\mathbb{S}_{-1}, \tau\right)$ by taking expectations over $\tau^{\prime}$. 
6. Iterate the procedures $2-5$ until the algorithm converges so that $\left\|\mathcal{W}^{(j)}\left(\mathbb{S}_{-1}, \tau\right)-\mathcal{W}^{(j-1)}\left(\mathbb{S}_{-1}, \tau\right)\right\|<$ $\epsilon,\left\|\mathcal{V}^{(j)}\left(\mathbb{S}_{-1}, \tau\right)-\mathcal{V}^{(j-1)}\left(\mathbb{S}_{-1}, \tau\right)\right\|<\epsilon$ and $\left\|\mathcal{K}^{(j)}\left(\mathbb{S}_{-1}, \tau\right)-\mathcal{K}^{(j-1)}\left(\mathbb{S}_{-1}, \tau\right)\right\|<\epsilon$ hold

Quasi-linear model The policy functions for the quasi-linear model are solved using the same algorithm above. As the model is approximated at the first order, higher-order price dispersion ceases to exist. Then, the vector of endogenous state variables is reduced to $\mathbb{S}_{-1} \equiv\left(\tilde{Y}_{-1}, \tilde{\Pi}_{-1}, \tilde{R}_{-1}^{*}\right)$. The regime-specific monetary policy rule is given by

$$
\begin{aligned}
& h_{R, 1}\left(\mathbb{S}_{-1}, \tau\right)=\phi_{r} \tilde{R}_{-1}^{*}+\left(1-\phi_{r}\right)\left(\phi_{\pi} h_{\Pi, 1}\left(\mathbb{S}_{-1}, \tau\right)+\phi_{y} h_{Y, 1}\left(\mathbb{S}_{-1}, \tau\right)\right)+\varepsilon_{r}, \\
& h_{R, 2}\left(\mathbb{S}_{-1}, \tau\right)=-\log \bar{R} .
\end{aligned}
$$

Given the processes of $\tau$, the regime-specific functions in each regime $i=1,2$ satisfy the following equilibrium conditions:

$$
\begin{aligned}
h_{Y, i}\left(\mathbb{S}_{-1}, \tau\right) & =\sum_{\tau^{\prime}} \Phi\left(\tau^{\prime} \mid \tau\right)\left\{\frac{1}{1+\gamma} h_{Y}\left(\mathbb{S}, \tau^{\prime}\right)+\frac{1-\gamma}{\sigma(1+\gamma)} h_{\Pi}\left(\mathbb{S}, \tau^{\prime}\right)\right\} \\
& +\frac{\gamma}{1+\gamma} \tilde{Y}_{-1}-\frac{1-\gamma}{\sigma(1+\gamma)}\left(h_{R, i}\left(\mathbb{S}_{-1}, \tau\right)-\tilde{d}\right), \\
h_{\Pi, i}\left(\mathbb{S}_{-1}, \tau\right) & =\frac{\beta}{1+\beta \iota} \sum_{\tau^{\prime}} \Phi\left(\tau^{\prime} \mid \tau\right) h_{\Pi}\left(\mathbb{S}, \tau^{\prime}\right)+\frac{\iota}{1+\beta \iota} \tilde{\Pi}_{-1} \\
& +\frac{(1-\xi)(1-\xi \beta)}{\xi(1+\beta \iota)}\left[\frac{\sigma}{1-\gamma} h_{Y, i}\left(\mathbb{S}_{-1}, \tau\right)-\frac{\sigma \gamma}{1-\gamma} \tilde{Y}_{-1}-\tilde{A}\right] .
\end{aligned}
$$

We define $\mathcal{W}_{i}\left(\mathbb{S}_{-1}, \tau\right) \equiv h_{Y, i}\left(\mathbb{S}_{-1}, \tau\right)$ and $\mathcal{V}_{i}\left(\mathbb{S}_{-1}, \tau\right) \equiv \sum_{\tau^{\prime}} \Phi\left(\tau^{\prime} \mid \tau\right) h_{\Pi}\left(h_{i}\left(\mathbb{S}_{-1}, \tau\right), \tau^{\prime}\right)$. Given $\mathcal{W}_{i}^{(j-1)}\left(\mathbb{S}_{-1}, \tau\right)$ and $\mathcal{V}_{i}^{(j-1)}\left(\mathbb{S}_{-1}, \tau\right)$, we have

$$
\begin{aligned}
h_{\Pi, i}\left(\mathbb{S}_{-1}, \tau\right) & =\frac{\beta}{1+\beta \iota} \mathcal{V}_{i}^{(j-1)}\left(\mathbb{S}_{-1}, \tau\right)+\frac{\iota}{1+\beta \iota} \tilde{\Pi}_{-1} \\
& +\frac{(1-\xi)(1-\xi \beta)}{\xi(1+\beta \iota)}\left[\frac{\sigma}{1-\gamma} \mathcal{W}_{i}^{(j-1)}\left(\mathbb{S}_{-1}, \tau\right)-\frac{\sigma \gamma}{1-\gamma} \tilde{Y}_{-1}-\tilde{A}\right] \\
h_{Y, i}\left(\mathbb{S}, \tau^{\prime}\right) & =\mathcal{W}_{i}^{(j-1)}\left(\mathbb{S}_{-1}, \tau\right) .
\end{aligned}
$$

There is a one-to-one mapping from $\mathcal{W}_{i}^{(j-1)}\left(\mathbb{S}_{-1}, \tau\right)$ and $\mathcal{V}_{i}^{(j-1)}\left(\mathbb{S}_{-1}, \tau\right)$ to $h_{\Pi, i}\left(\mathbb{S}_{t-1}, \tau_{t}\right)$ and $h_{Y, i}\left(\mathbb{S}_{-1}, \tau\right)$. Then the values of $\mathcal{W}_{i}^{(j)}\left(\mathbb{S}_{-1}, \tau\right)$ and $\mathcal{V}_{i}^{(j)}\left(\mathbb{S}_{-1}, \tau\right)$ are updated as follows:

$$
\begin{aligned}
\mathcal{W}_{i}^{(j)}\left(\mathbb{S}_{-1}, \tau\right) & =\sum_{\tau^{\prime}} \Phi\left(\tau^{\prime} \mid \tau\right)\left\{\frac{1}{1+\gamma} \hat{h}_{Y}\left(h_{i}\left(\mathbb{S}_{-1}, \tau\right), \tau^{\prime}\right)+\frac{1-\gamma}{\sigma(1+\gamma)} \hat{h}_{\Pi}\left(h_{i}\left(\mathbb{S}_{-1}, \tau\right), \tau^{\prime}\right)\right\} \\
& +\frac{\gamma}{1+\gamma} \tilde{Y}_{-1}-\frac{1-\gamma}{\sigma(1+\gamma)}\left(h_{R, i}\left(\mathbb{S}_{-1}, \tau\right)-\tilde{d}\right), \\
\mathcal{V}_{i}^{(j)}\left(\mathbb{S}_{-1}, \tau\right) & =\sum_{\tau^{\prime}} \Phi\left(\tau^{\prime} \mid \tau\right) \hat{h}_{\Pi}\left(h_{i}\left(\mathbb{S}_{-1}, \tau\right), \tau^{\prime}\right),
\end{aligned}
$$


where $\hat{h}_{\Pi}\left(\mathbb{S} ; \tau^{\prime}\right)$ and $\hat{h}_{Y}\left(\mathbb{S} ; \tau^{\prime}\right)$ are evaluated from the estimated values of $\hat{\mathcal{W}}\left(\mathbb{S} ; \tau^{\prime}\right) \approx \mathcal{W}^{(j-1)}\left(\mathbb{S}, \tau^{\prime}\right)$ and $\hat{\mathcal{V}}\left(\mathbb{S} ; \tau^{\prime}\right) \approx \mathcal{V}^{(j-1)}\left(\mathbb{S}, \tau^{\prime}\right)$.

Accuracy of the solutions As we rely on approximation to solve for the policy functions $h\left(\mathbb{S}_{-1}, \tau\right)$, we assess the accuracy of the solutions, following Maliar and Maliar (2015). In each experiment, equilibrium paths are simulated for 40,000 periods ( $N=200$ Monte Carlo replications with a sample size of $T=200$ observations). For each sample of $\left\{\left(\mathbb{S}_{t-1}, \tau_{t}\right)\right\}_{t=1, . ., T}^{i}$ where $i=1, \ldots, N$, we compute the residual functions:

$$
\begin{aligned}
\mathcal{R}_{1}\left(\mathbb{S}_{t-1}, \tau_{t}\right) & =1-\left[\beta d^{-1} h_{R}\left(\mathbb{S}_{t-1}, \tau_{t}\right) \sum_{\tau^{\prime}}\left\{\frac{\Phi\left(\tau^{\prime} \mid \tau_{t}\right)}{\hat{\mathcal{W}}\left(h\left(\mathbb{S}_{t-1}, \tau_{t}\right) ; \tau^{\prime}\right) \hat{h}_{\Pi}\left(h\left(\mathbb{S}_{t-1}, \tau_{t}\right) ; \tau^{\prime}\right)}\right\}\right] \mathcal{W}\left(\mathbb{S}_{t-1}, \tau_{t}\right), \\
\mathcal{R}_{2}\left(\mathbb{S}_{t-1}, \tau_{t}\right) & =1-\sum_{\tau^{\prime}}\left\{\left[\left(\frac{\hat{h}_{\Pi}\left(h\left(\mathbb{S}_{t-1}, \tau_{t}\right) ; \tau^{\prime}\right)}{\Pi}\right)\left(\frac{h_{\Pi}\left(\mathbb{S}_{t-1}, \tau_{t}\right)}{\Pi}\right)^{-\iota}\right]^{\theta} \frac{\hat{h}_{Y}\left(h\left(\mathbb{S}_{t-1}, \tau_{t}\right) ; \tau^{\prime}\right)}{h_{Y}\left(\mathbb{S}_{t-1}, \tau_{t}\right)}\right. \\
& \left.\times\left(\theta A^{\prime-1}+d^{\prime-1} \beta \xi \hat{\mathcal{V}}\left(h\left(\mathbb{S}_{t-1}, \tau_{t}\right) ; \tau^{\prime}\right)\right) \Phi\left(\tau^{\prime} \mid \tau_{t}\right)\right\} / \mathcal{V}\left(\mathbb{S}_{t-1}, \tau_{t}\right), \\
\mathcal{R}_{3}\left(\mathbb{S}_{t-1}, \tau_{t}\right) & =1-\sum_{\tau^{\prime}}\left\{\left[\left(\frac{\hat{h}_{\Pi}\left(h\left(\mathbb{S}_{t-1}, \tau_{t}\right) ; \tau^{\prime}\right)}{\Pi}\right)\left(\frac{h_{\Pi}\left(h\left(\mathbb{S}_{t-1}, \tau_{t}\right), \tau^{\prime}\right)}{\Pi}\right)^{-\iota}\right]^{\theta-1} \frac{\hat{h}_{Y}\left(h\left(\mathbb{S}_{t-1}, \tau_{t}\right) ; \tau^{\prime}\right)}{h_{Y}\left(\mathbb{S}_{t-1}, \tau_{t}\right)}\right. \\
& \left.\times\left((\theta-1) / \hat{\mathcal{V}}\left(h\left(\mathbb{S}_{t-1}, \tau_{t}\right) ; \tau^{\prime}\right)+d^{\prime-1} \beta \xi \hat{\mathcal{K}}\left(h\left(\mathbb{S}_{t-1}, \tau_{t}\right) ; \tau^{\prime}\right)\right) \Phi\left(\tau^{\prime} \mid \tau_{t}\right)\right\} / \mathcal{K}\left(\mathbb{S}_{t-1}, \tau_{t}\right) .
\end{aligned}
$$

Then, we calculate the mean and maximum of the residuals $\left\{\mathcal{R}_{j}\left(\mathbb{S}_{t-1}, \tau_{t}\right)\right\}^{i}$ across different samples $i=1, \ldots, N$ and residuals $j=1, \ldots, J{ }^{21}$

The second and third columns of Table 5 (Equilibrium paths) show the accuracy of each solution for the nonlinear model with the ZLB (Baseline), the nonlinear model without the ZLB (No ZLB in DGP), the quasi-linear model with the ZLB (Quasi-linear DGP), and the two models with different parameter values from the baseline (Case of low $\overline{r r}$ and Case of large $\sigma_{d}$ ). The maximum size of residuals is about $0.3 \%$ in the baseline model $\left(10^{-2.527}\right)$, and it is similar in the cases of low $\overline{r r}$ and

\footnotetext{
${ }^{21}$ In the quasi-linear model, the residual functions are

$$
\begin{aligned}
\mathcal{R}_{1}\left(\mathbb{S}_{t-1}, \tau_{t}\right) & =h_{Y}\left(\mathbb{S}_{t-1}, \tau_{t}\right)-\frac{\gamma}{1+\gamma} \tilde{Y}_{t-1}+\frac{1-\gamma}{\sigma(1+\gamma)}\left(h_{R}\left(\mathbb{S}_{t-1}, \tau_{t}\right)-\tilde{d}_{t}\right) \\
& -\sum_{\tau^{\prime}} \Phi\left(\tau^{\prime} \mid \tau_{t}\right)\left\{\frac{1}{1+\gamma} \hat{h}_{Y}\left(h\left(\mathbb{S}_{t-1}, \tau_{t}\right) ; \tau^{\prime}\right)+\frac{1-\gamma}{\sigma(1+\gamma)} \hat{h}_{\Pi}\left(h\left(\mathbb{S}_{t-1}, \tau_{t}\right) ; \tau^{\prime}\right)\right\} \\
\mathcal{R}_{2}\left(\mathbb{S}_{t-1}, \tau_{t}\right) & =h_{\Pi}\left(\mathbb{S}_{t-1}, \tau_{t}\right)-\frac{\iota}{1+\beta \iota} \tilde{\Pi}_{t-1}-\frac{\beta}{1+\beta \iota} \sum_{\tau^{\prime}} \Phi\left(\tau^{\prime} \mid \tau_{t}\right) \hat{h}_{\Pi}\left(h\left(\mathbb{S}_{t-1}, \tau_{t}\right) ; \tau^{\prime}\right) \\
& -\frac{(1-\xi)(1-\xi \beta)}{\xi(1+\beta \iota)}\left[\frac{\sigma}{1-\gamma} h_{Y}\left(\mathbb{S}_{t-1}, \tau_{t}\right)-\frac{\sigma \gamma}{1-\gamma} \tilde{Y}_{t-1}-\tilde{A}_{t}\right] .
\end{aligned}
$$
}


large $\sigma_{d}$. This result suggests that our solution method is very accurate even in the presence of the ZLB, compared with previous studies such as Fernández-Villaverde, Gordon, Guerrón-Quintana, and Rubio-Ramírez (2015) and Maliar and Maliar (2015). ${ }^{22}$ The case of the quasi-linear model is even more accurate than the baseline case, although it is less accurate than that of the model without the ZLB; the maximum residuals are about $0.06 \%$ and $<0.01 \%\left(10^{-3.195}\right.$ and $\left.10^{-4.570}\right)$, respectively.

We also evaluate the accuracy of the solutions based on 40,000 random points from uniform distributions over the entire state space. The last two columns of Table 5 (Entire state space) demonstrate the same tendency across the cases, although the errors are slightly larger (but acceptable).

\footnotetext{
${ }^{22}$ Fernández-Villaverde, Gordon, Guerrón-Quintana, and Rubio-Ramírez (2015), who use a Smolyak algorithm and the same level of approximation, $\mu=2$, report that the mean and maximum of the Euler equation error $\left(\mathcal{R}_{1}\left(\mathbb{S}_{t-1}, \tau_{t}\right)\right.$ in this paper) are $10^{-3.256}$ and $10^{-1.648}$. Maliar and Maliar (2015) show that the mean and maximum errors across all equilibrium conditions (as in this paper) are $10^{-3.65}$ and $10^{-1.81}$ in the case with the ZLB and a third-degree polynomial solution with the EDS ( $\varepsilon$-distinguishable set) grid.
} 


\section{References}

Basu, Susanto, and Brent Bundick, 2012. "Uncertainty Shocks in a Model of Effective Demand," NBER Working Paper 18420, National Bureau of Economic Research.

van Binsbergen, Jules H., Fernández-Villaverde, Koijen, Ralph S. J., and Juan F. Rubio-Ramírez, 2012. "The Term Structure of Interest Rates in a DSGE Model with Recursive Preferences." Journal of Monetary Economics, 59(7), 634-648.

Bodenstein, Martin, Luca Guerrieri, and Christopher J. Gust, 2013. "Oil Shocks and the Zero Bound on Nominal Interest Rates." Journal of International Money and Finance, 32, 941-967.

Braun, R. Anton, Lena M. Körber, and Yuichiro Waki, 2012. "Some Unpleasant Properties of LogLinearized Solutions When the Nominal Rate is Zero." Working Paper 2012-05a, Federal Reserve Bank of Atlanta.

Calvo, Guillermo A., 1983. "Staggered Prices in a Utility-Maximizing Framework." Journal of Monetary Economics, 12(3), 383-398.

Christiano, Lawrence, Martin Eichenbaum, and Sergio Rebelo, 2011. "When Is the Government Spending Multiplier Large?" Journal of Political Economy, 119(1), 78-121.

Eggertsson, Gauti B., and Michael Woodford, 2003. "The Zero Bound on Interest Rates and Optimal Monetary Policy." Brookings Papers on Economic Activity, 34(1), 139-235.

Erceg, Christopher J., and Jesper Lindé, 2014. "Is There a Fiscal Free Lunch in a Liquidity Trap." Journal of European Economic Association, 12(1), 73-107.

Fernández-Villaverde, Jesús, and Juan F. Rubio-Ramírez, 2005. "Estimating Dynamic Equilibrium Economies: Linear Versus Nonlinear Likelihood." Journal of Applied Econometrics, 20(7), 891910.

Fernández-Villaverde, Jesús, and Juan F. Rubio-Ramírez, 2007. "Estimating Macroeconomic Models: A Likelihood Approach." Review of Economic Studies, 74(4), 1059-1087.

Fernández-Villaverde, Jesús, Juan F. Rubio-Ramírez, and Manuel S. Santos, 2006. "Convergence Properties of the Likelihood of Computed Dynamic Models." Econometrica, 74(1), 93-119. 
Fernández-Villaverde, Jesús, Grey Gordon, Pablo A. Guerrón-Quintana, and Juan Rubio-Ramírez, 2015. "Nonlinear Adventures at the Zero Lower Bound." Journal of Economic Dynamics and Control, 57, 182-204.

Gavin, William T., Benjamin D. Keen, Alexander Richter, and Nathaniel Throckmorton, 2015. "The Zero Lower Bound, the Dual Mandate, and Unconventional Dynamics." Journal of Economic Dynamics and Control, 55, 14-38.

Gust, Christopher, David Lopez-Salido, and Matthew E. Smith, 2012. "The Empirical Implications of the Interest-Rate Lower Bound." Finance and Economics Discussion Series 2012-83, Board of Governors of the Federal Reserve System.

Hirose, Yasuo, and Atsushi Inoue, 2015. "The Zero Lower Bound and Parameter Bias in an Estimated DSGE Model." Journal of Applied Econometrics, forthcoming.

Judd, Kenneth L., Lilia Maliar, Serguei Maliar, and Rafael Valero, 2015. "Smolyak Method for Solving Dynamic Economic Models: Lagrange Interpolation, Anisotropic Grid and Adaptive Domain." Journal of Economic Dynamics and Control, 44, 92-123.

Justiniano, Alejandro, Giorgio E. Primiceri, and Andrea Tambalotti, 2010. "Investment Shocks and Business Cycles." Journal of Monetary Economics, 57, 132-145.

Malin, Benjamin A., Dirk, Krueger, and Felix Kubler, 2011. "Solving the Multi-Country Real Business Cycle Model using a Smolyak-Collocation Method." Journal of Economic Dynamics and Control, 35(2), 229-239.

Lubik, Thomas A., and Frank Schorfheide, 2004. "Testing for Indeterminacy: An Application to U.S. Monetary Policy.” American Economic Review, 94(1), 190-217.

Maliar, Lilia, and Serguei Maliar, 2015. "Merging Simulation and Projection Aproaches to Solve High-Dimensional Problems with an Application to a New Keynesian Model." Quantitative Economics, 6, 1-47.

Nakata, Taisuke, 2013a. "Uncertainty at the Zero Lower Bound." Finance and Economics Discussion Series 2013-09, Board of Governors of the Federal Reserve System.

Nakata, Taisuke, 2013b. "Optimal Fiscal and Monetary Policy with Occasionally Binding Zero Bound Constraints." Finance and Economics Discussion Series 2013-40, Board of Governors of the Federal Reserve System. 
Ngo, Phuong V., 2014. "Optimal Discretionary Monetary Policy in a Micro-Founded Model with a Zero Lower Bound on Nominal Interest Rate." Journal of Economic Dynamics and Control, 45, $44-65$.

Rouwenhorst, K. Geert, 1995. "Asset Pricing Implications of Equilibrium Business Cycle Models." In: Cooley, Thomas F. (ed.), Frontiers of Business Cycle Research, 294-330. Princeton University Press, Princeton, NJ.

Smets, Frank, and Rafael Wouters, 2007. "Shocks and Frictions in US Business Cycles: A Bayesian DSGE Approach." American Economic Review, 97(3), 586-606.

Taylor, John B., 1993. "Discretion Versus Policy Rules in Practice." Carnegie-Rochester Conference Series on Public Policy, 39(1), 195-214. 


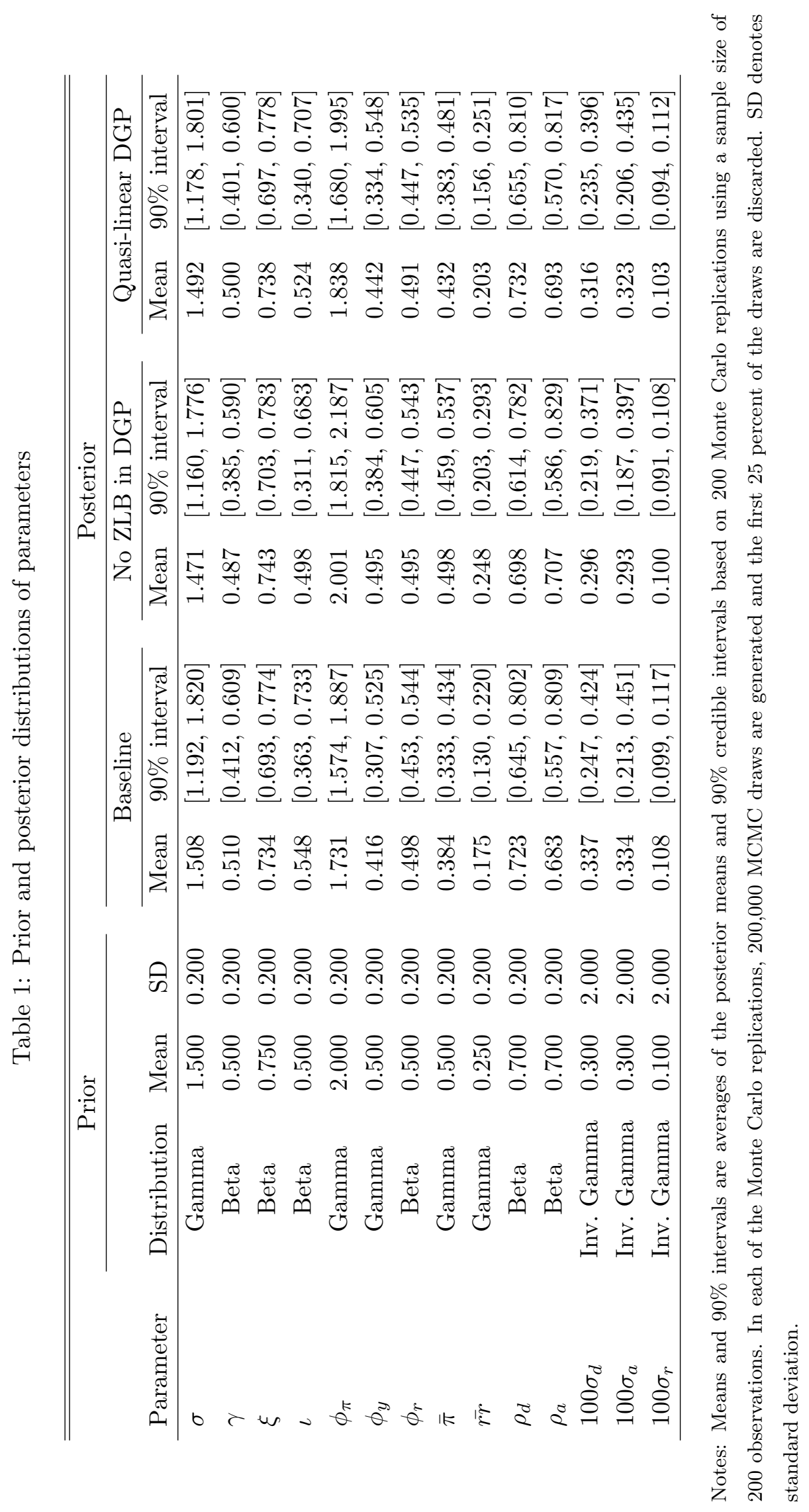


Table 2: Variance-covariance matrices of observed variables

\begin{tabular}{|c|c|c|c|}
\hline & Output & Inflation & Interest rate \\
\hline \multicolumn{4}{|c|}{ (1) Nonlinear model with true parameters (DGP) } \\
\hline Output & 0.090 & 0.038 & 0.069 \\
\hline Inflation & & 0.036 & 0.059 \\
\hline Interest rate & & & 0.129 \\
\hline \multicolumn{4}{|c|}{ (2) Linear model with true parameters } \\
\hline Output & 0.066 & 0.015 & 0.041 \\
\hline Inflation & & 0.017 & 0.032 \\
\hline Interest rate & & & 0.088 \\
\hline \multicolumn{4}{|c|}{ (3) Linear model with biased parameters } \\
\hline Output & 0.096 & 0.037 & 0.071 \\
\hline Inflation & & 0.037 & 0.063 \\
\hline Interest rate & & & 0.139 \\
\hline
\end{tabular}

Notes: In the case of the nonlinear model with the true parameters, the empirical variance-covariance matrix is calculated by an average of the sample variances and covariances based on 200 Monte Carlo replications with a sample size of 200 observations. In the cases of the linear models with the true and biased parameters, the theoretical variance-covariance matrices are computed by solving the discrete Lyapunov equations. The biased parameters are set at the posterior mean estimates in the baseline experiment shown in Table 1. 
Table 3: Posterior distributions of parameters in alternative experiments

\begin{tabular}{lcccccc}
\hline \hline & & \multicolumn{2}{c}{ Case of low $\bar{r} r$} & & \multicolumn{2}{c}{ Case of large $\sigma_{d}$} \\
\cline { 3 - 4 } \cline { 7 - 8 } Parameter & True value & Mean & $90 \%$ interval & & Mean & $90 \%$ interval \\
\hline$\sigma$ & 1.500 & 1.530 & {$[1.212,1.844]$} & & 1.528 & {$[1.209,1.842]$} \\
$\gamma$ & 0.500 & 0.521 & {$[0.424,0.619]$} & & 0.521 & {$[0.425,0.619]$} \\
$\xi$ & 0.750 & 0.733 & {$[0.693,0.773]$} & & 0.733 & {$[0.694,0.773]$} \\
$\iota$ & 0.500 & 0.570 & {$[0.388,0.754]$} & & 0.573 & {$[0.390,0.755]$} \\
$\phi_{\pi}$ & 2.000 & 1.614 & {$[1.456,1.771]$} & & 1.619 & {$[1.459,1.778]$} \\
$\phi_{y}$ & 0.500 & 0.376 & {$[0.263,0.487]$} & & 0.376 & {$[0.265,0.486]$} \\
$\phi_{r}$ & 0.500 & 0.514 & {$[0.466,0.562]$} & & 0.515 & {$[0.468,0.561]$} \\
$\bar{\pi}$ & 0.500 & 0.347 & {$[0.294,0.400]$} & & 0.352 & {$[0.299,0.405]$} \\
$\overline{r r}$ & $0.22 / 0.25$ & 0.138 & {$[0.094,0.182]$} & & 0.175 & {$[0.130,0.219]$} \\
$\rho_{d}$ & 0.700 & 0.721 & {$[0.644,0.800]$} & & 0.716 & {$[0.638,0.794]$} \\
$\rho_{a}$ & 0.700 & 0.672 & {$[0.544,0.800]$} & & 0.670 & {$[0.543,0.799]$} \\
$100 \sigma_{d}$ & $0.30 / 0.33$ & 0.347 & {$[0.251,0.440]$} & & 0.382 & {$[0.277,0.484]$} \\
$100 \sigma_{a}$ & 0.300 & 0.355 & {$[0.225,0.481]$} & & 0.356 & {$[0.226,0.481]$} \\
$100 \sigma_{r}$ & 0.100 & 0.115 & {$[0.105,0.124]$} & & 0.118 & {$[0.108,0.128]$} \\
\hline
\end{tabular}

Notes: In the case of low $\overline{r r}$, the true value of $\overline{r r}$ is 0.22 . In the case of large $\sigma_{d}$, the true value of $100 \sigma_{d}$ is 0.33 . Means and $90 \%$ intervals are averages of the posterior means and 90\% credible intervals based on 200 Monte Carlo replications using a sample size of 200 observations. In each of the Monte Carlo replications, 200,000 MCMC draws are generated and the first 25 percent of the draws are discarded. 
Table 4: Variance-covariance matrices of observed variables in alternative experiments

\begin{tabular}{|c|c|c|c|c|c|c|}
\hline & \multicolumn{3}{|c|}{ Case of low $\overline{r r}$} & \multicolumn{3}{|c|}{ Case of large $\sigma_{d}$} \\
\hline & Output & Inflation & Interest rate & Output & Inflation & Interest rate \\
\hline \multicolumn{7}{|c|}{ (1) Nonlinear model with true parameters (DGP) } \\
\hline Output & 0.101 & 0.047 & 0.075 & 0.118 & 0.055 & 0.089 \\
\hline Inflation & & 0.044 & 0.065 & & 0.048 & 0.072 \\
\hline Interest rate & & & 0.130 & & & 0.146 \\
\hline \multicolumn{7}{|c|}{ (2) Linear model with true parameters } \\
\hline Output & 0.066 & 0.015 & 0.041 & 0.076 & 0.020 & 0.053 \\
\hline Inflation & & 0.017 & 0.032 & & 0.019 & 0.038 \\
\hline Interest rate & & & 0.088 & & & 0.103 \\
\hline \multicolumn{7}{|c|}{ (3) Linear model with biased parameters } \\
\hline Output & 0.108 & 0.045 & 0.076 & 0.124 & 0.054 & 0.091 \\
\hline Inflation & & 0.045 & 0.069 & & 0.049 & 0.077 \\
\hline Interest rate & & & 0.143 & & & 0.160 \\
\hline
\end{tabular}

Notes: In the case of the nonlinear model with the true parameters, the empirical variance-covariance matrix is calculated by an average of the sample variances and covariances based on 200 Monte Carlo replications with a sample size of 200 observations. In the cases of the linear models with the true and biased parameters, the theoretical variance-covariance matrices are computed by solving the discrete Lyapunov equations. The biased parameters are set at the posterior mean estimates in the alternative experiments shown in Table 3.

Table 5: Accuracy of solutions

\begin{tabular}{lccccc}
\hline \hline & \multicolumn{2}{c}{ Equilibrium paths } & & \multicolumn{2}{c}{ Entire state space } \\
\cline { 2 - 3 } \cline { 5 - 6 } & $\log _{10} L_{1}$ & $\log _{10} L_{\infty}$ & & $\log _{10} L_{1}$ & $\log _{10} L_{\infty}$ \\
\hline Baseline & -3.508 & -2.527 & & -2.951 & -1.780 \\
No ZLB in DGP & -5.045 & -4.570 & & -4.831 & -4.008 \\
Quasi-linear DGP & -4.160 & -3.195 & & -3.522 & -2.456 \\
Case of low $\bar{r} r$ & -3.477 & -2.531 & & -2.961 & -1.770 \\
Case of large $\sigma_{d}$ & -3.453 & -2.532 & & -2.948 & -1.784 \\
\hline
\end{tabular}

Note: $L_{1}$ and $L_{\infty}$ are the mean and maximum of the absolute residuals across all the equilibrium conditions based on 40,000 points of $\left(\mathbb{S}_{-1}, \tau\right)$ on the equilibrium paths and 40,000 random points from uniform distributions over the entire state space, respectively. 
Figure 1: Policy functions
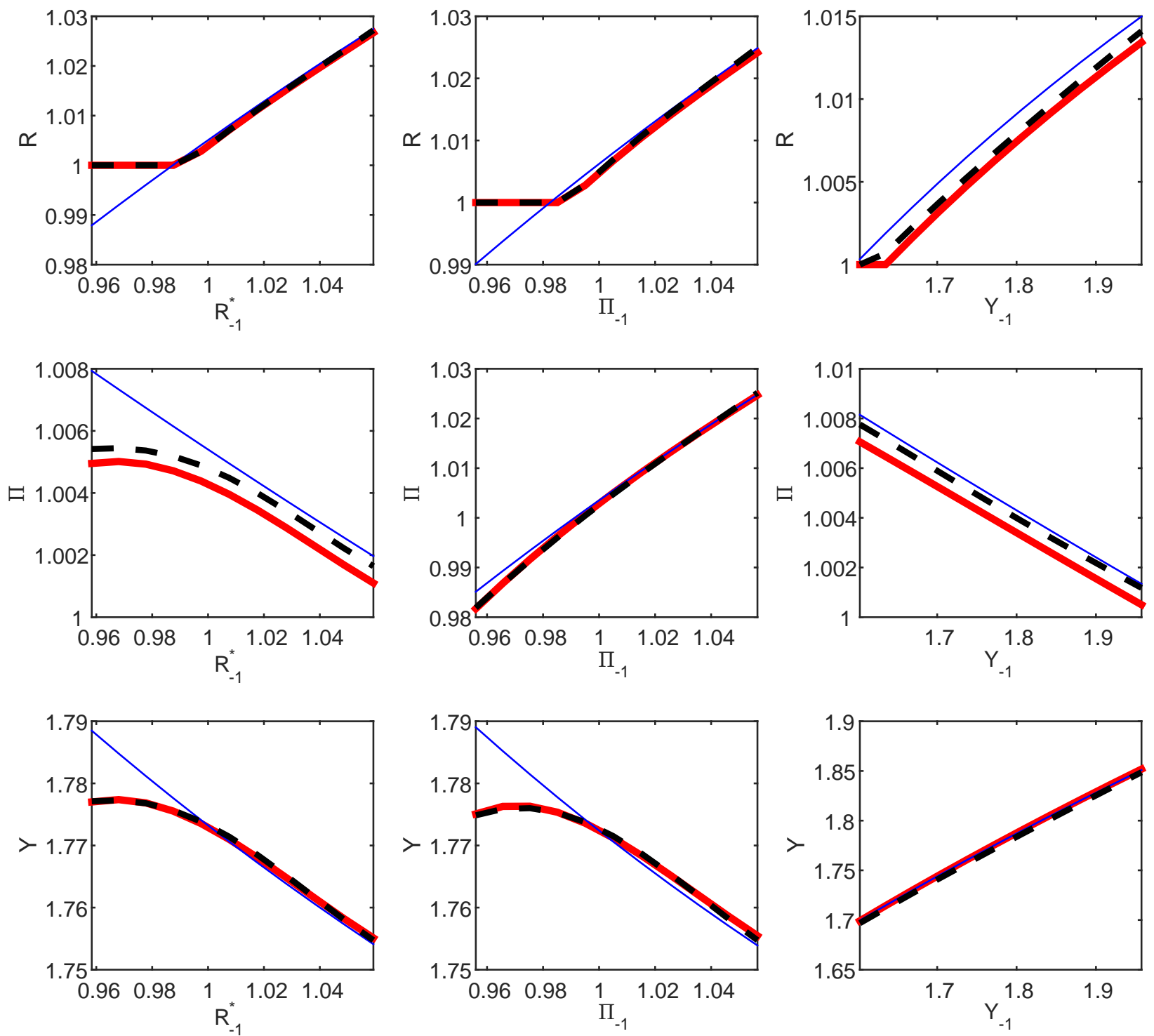

Notes: The figure shows the policy functions of output, inflation, and the nominal interest rate for the range of the state variables shown on each horizontal axis. The thick solid, thin solid, and dashed lines represent the policy functions of the nonlinear model with the ZLB, the nonlinear model without the ZLB, and the quasi-linear model with the ZLB, respectively. 
Figure 2: Impulse responses in the baseline experiment

(1) Discount factor shock
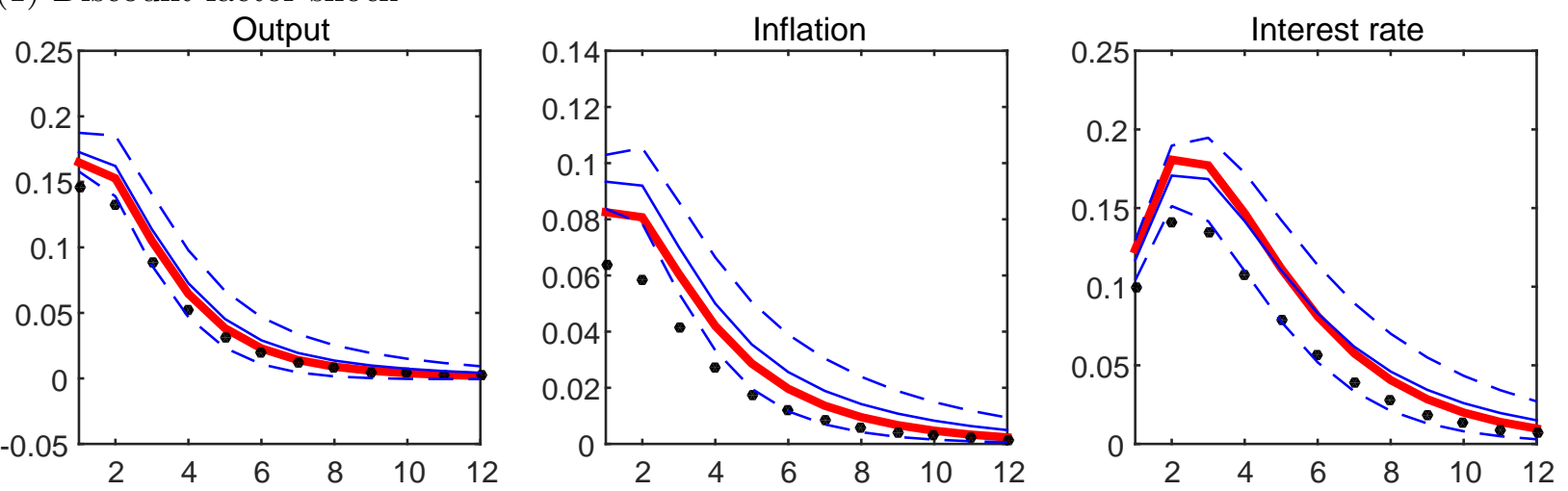

(2) Productivity shock
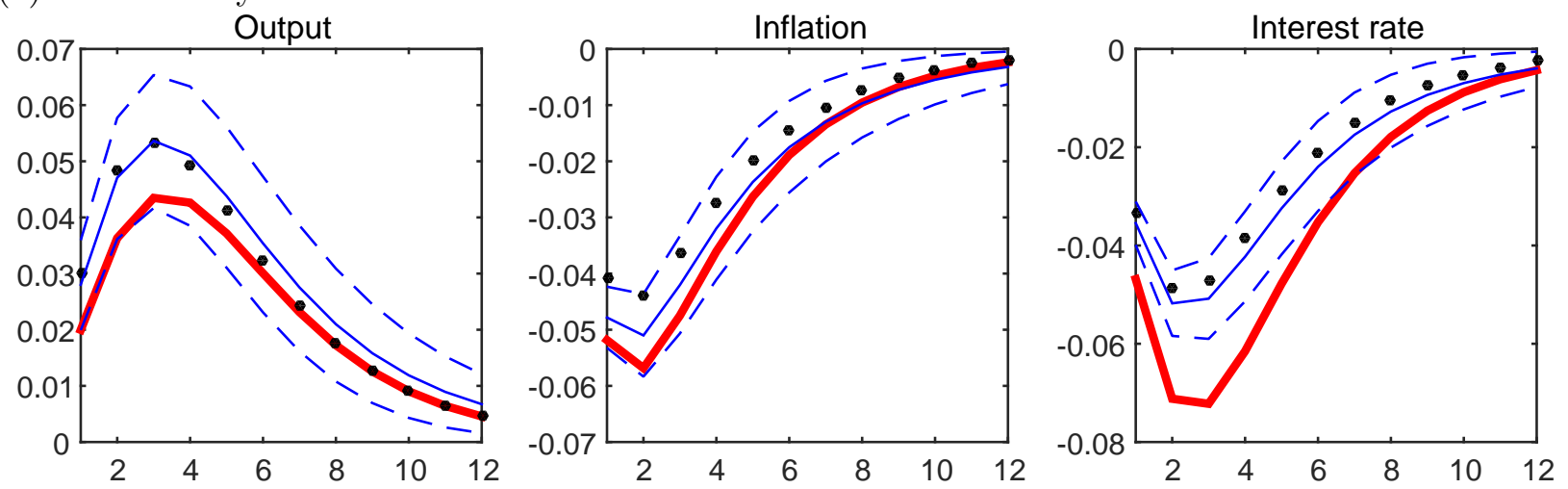

(3) Monetary policy shock
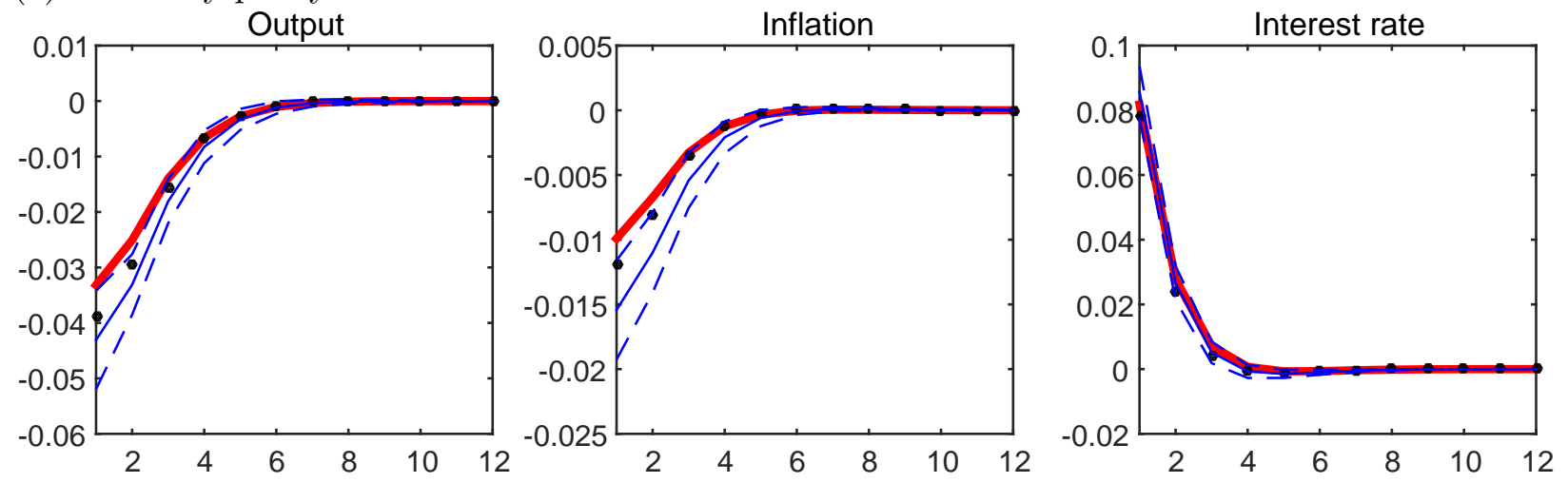

Notes: The figure shows the impulse responses of output, inflation, and the nominal interest rate (in terms of percentage deviations from the steady state) to one standard deviation shocks to the discount factor, productivity, and monetary policy. The thick solid lines represent the true responses, the dots are the responses of the linearized model with the true parameters, and the thin solid and dashed lines are the averages of the posterior means and 90\% credible intervals for the estimated responses, respectively, where the averages are taken over 200 Monte Carlo replications. 
Figure 3: Impulse responses in the case of low $\overline{r r}$

(1) Discount factor shock
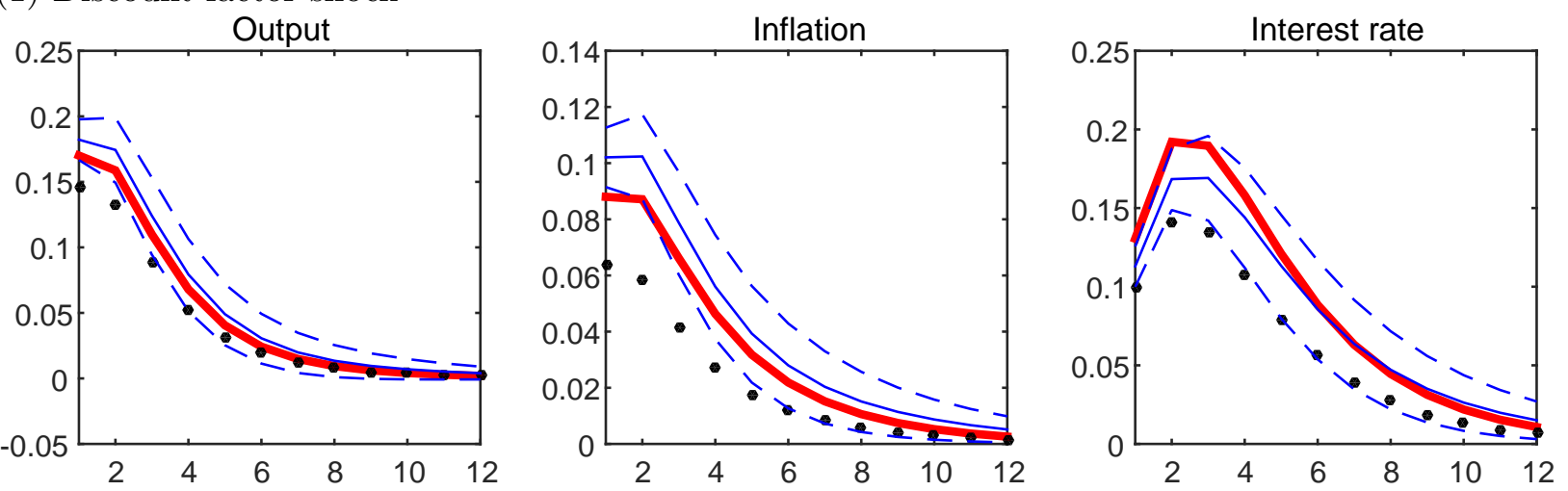

(2) Productivity shock
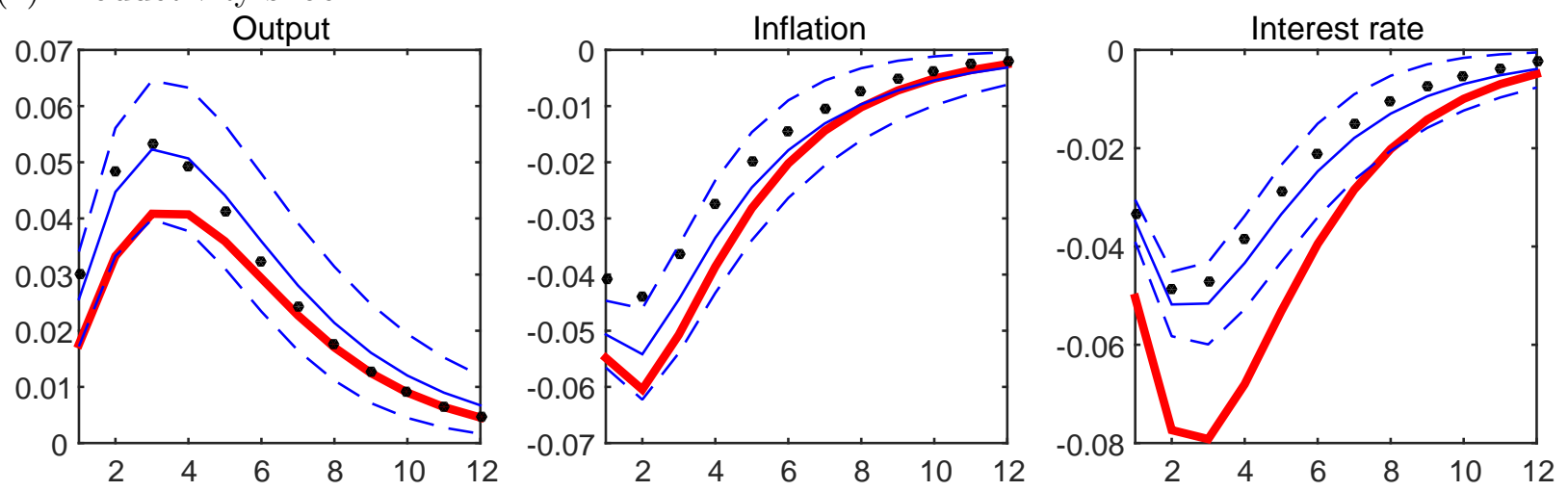

(3) Monetary policy shock
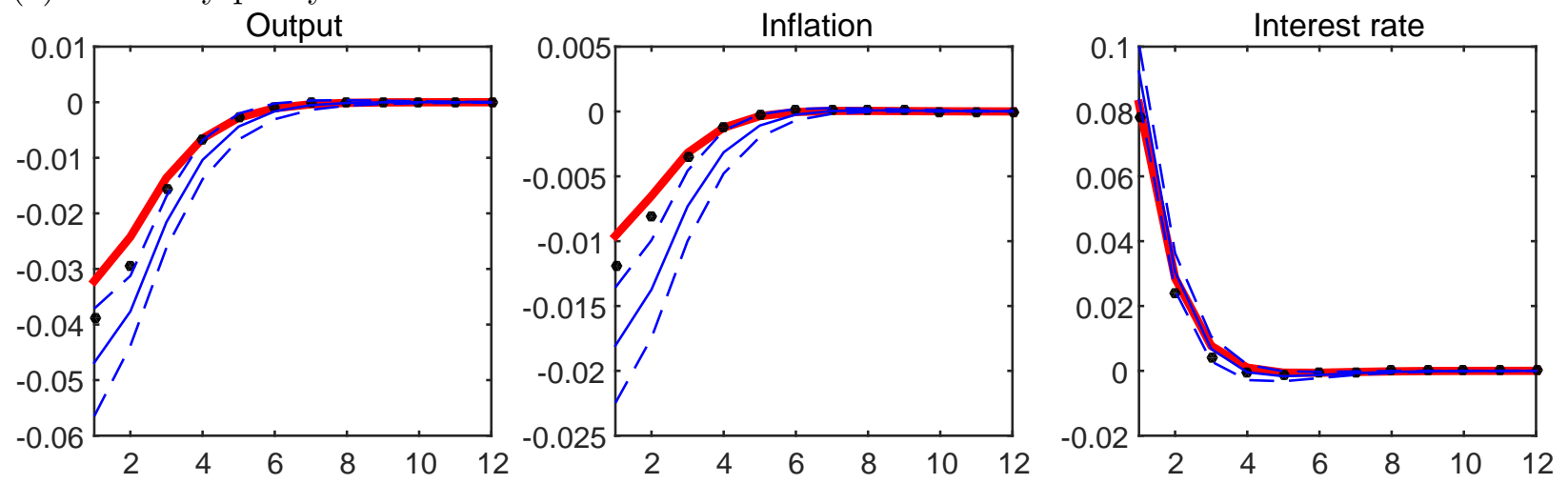

Notes: The figure shows the impulse responses of output, inflation, and the nominal interest rate (in terms of percentage deviations from the steady state) to one standard deviation shocks to the discount factor, productivity, and monetary policy. The thick solid lines represent the true responses, the dots are the responses of the linearized model with the true parameters, and the thin solid and dashed lines are the averages of the posterior means and 90\% credible intervals for the estimated responses, respectively, where the averages are taken over 200 Monte Carlo replications. 
Figure 4: Impulse responses in the case of large $\sigma_{d}$

(1) Discount factor shock
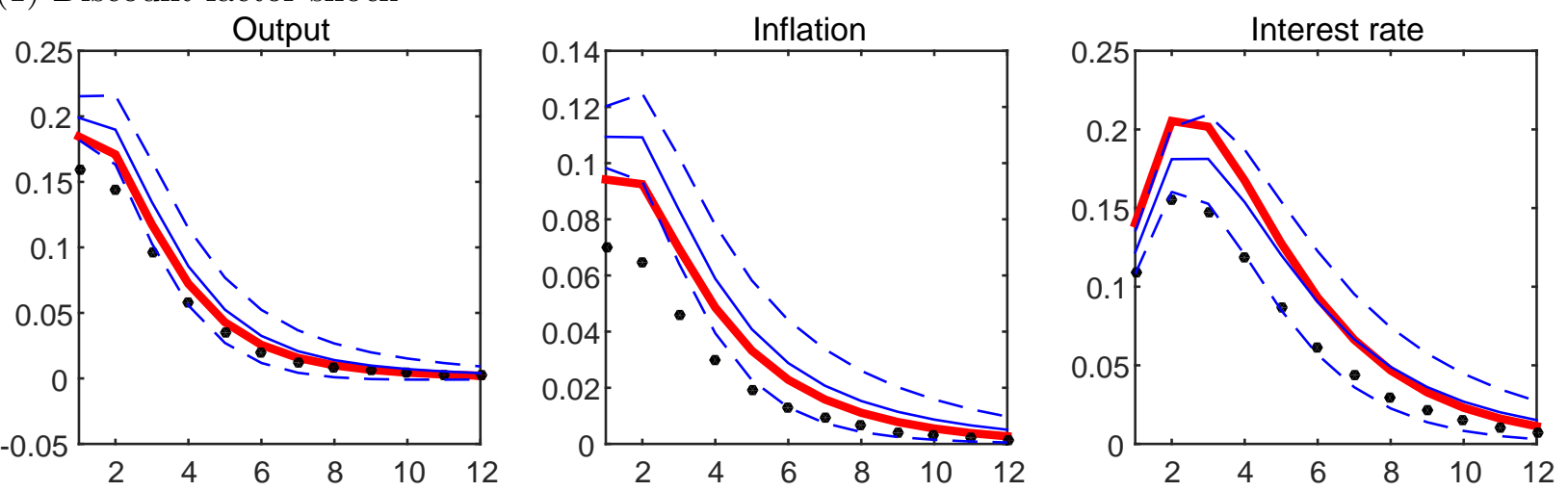

(2) Productivity shock
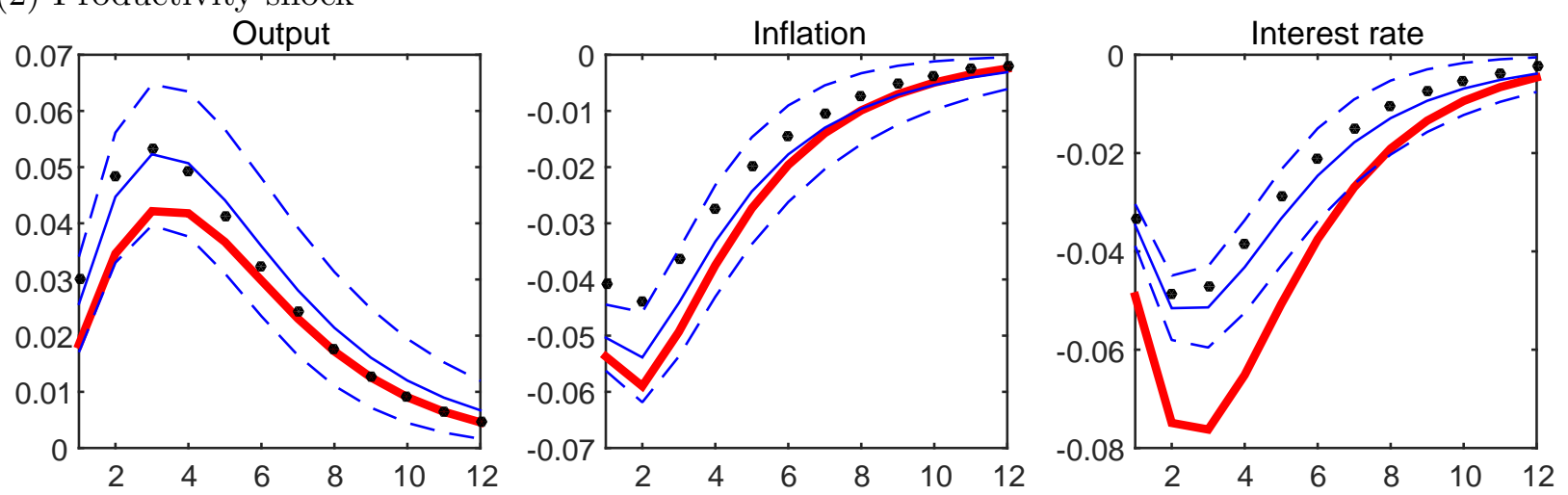

(3) Monetary policy shock
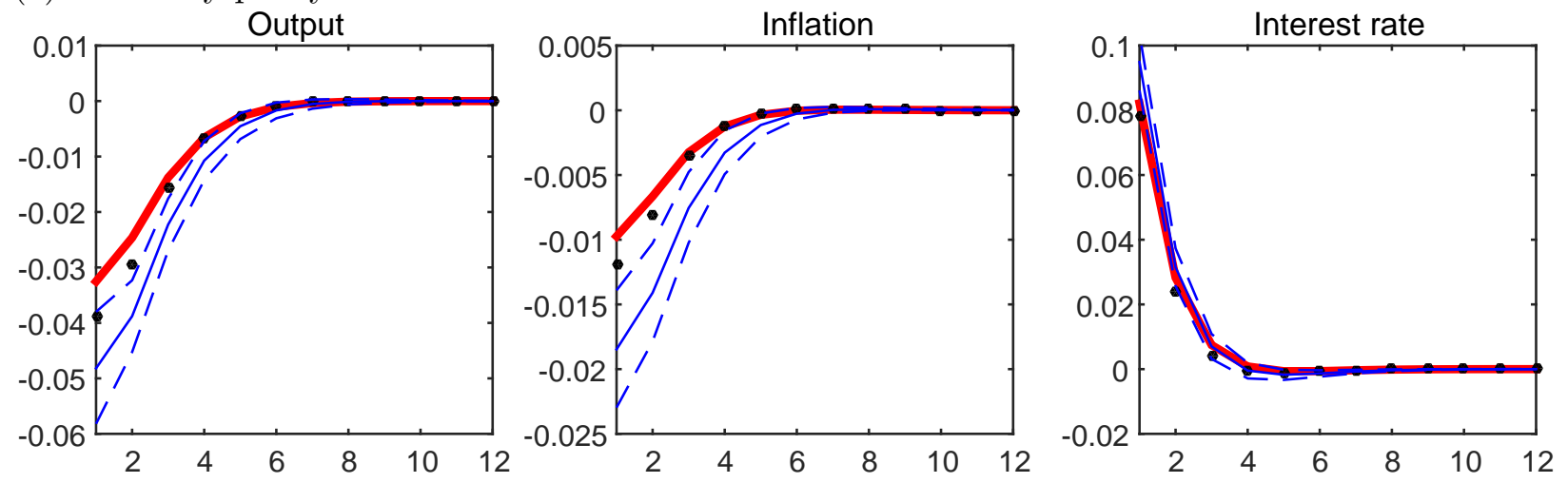

Notes: The figure shows the impulse responses of output, inflation, and the nominal interest rate (in terms of percentage deviations from the steady state) to one standard deviation shocks to the discount factor, productivity, and monetary policy. The thick solid lines represent the true responses, the dots are the responses of the linearized model with the true parameters, and the thin solid and dashed lines are the averages of the posterior means and 90\% credible intervals for the estimated responses, respectively, where the averages are taken over 200 Monte Carlo replications. 